\title{
Evaluación de variables meteorológicas modeladas para determinar el clima de ciudades costeras argentinas
}

\author{
Assessment of modelled meteorological variables to determine \\ the climate of coastal cities in Argentina
}

\author{
Federico Ferrelli $1^{* *}$ \\ María Luján Bustos ${ }^{2}$ \\ Gerardo M. E Perillo ${ }^{3}$ (i) \\ María Cintia Piccolo ${ }^{4}$
}

\section{Resumen}

El conocimiento del clima de las ciudades costeras es importante para el ordenamiento del espacio urbano. Para su estudio, se necesitan series superiores a 30 años que suelen ser escasas o inconsistentes. En este contexto, los modelos numéricos favorecen la generación de datos meteorológicos. Por ello, el objetivo de este estudio fue evaluar la precisión y ajuste entre datos de temperatura del aire, humedad relativa y precipitación obtenidos con el Reanalysis NCEP/NCAR y observaciones in situ para ocho ciudades costeras marinas y fluviales de Argentina localizadas en distintas regiones climáticas. Para ello, se compararon series meteorológicas del período 1960-2019 y las provenientes del Reanalysis. Ambas se evaluaron considerando distintas escalas espacio-temporales, utilizando diferentes métodos estadísticos. Los mismos favorecieron el conocimiento del ajuste entre las series en periodos normales, pero también se analizaron los valores extremos de las series a partir de una regresión por cuantiles. La temperatura del aire se ajustó mejor en las escalas climática (60 años), mensual y modelada. La humedad relativa tuvo más correlación en la escala estacional, en invierno y otoño, excepto para las ciudades de clima cálido y húmedo. Finalmente, en las precipitaciones el ajuste fue mejor en todas las escalas temporales para los climas templados y áridos. Los resultados generados representan una base de datos esencial para la toma de decisiones orientada a mejorar la calidad de vida de la población y el ambiente costero.

Palabras clave: Reanalysis NCEP/NCAR; datos in situ; ciudades costeras; Argentina.

1 Instituto Argentino de Oceanografía (IADO), Universidad Nacional del Sur (UNS)-CONICET, Bahía Blanca-Argentina. Teléfono: (54) (291) (4861112). B8000BFW, Bahía Blanca, Argentina. Florida 8000 (Camino La Carrindanga km 7,5) Complejo CCT CONICET Bahía Blanca, Edificio El.

Departamento de Geografía y Turismo, Universidad Nacional del Sur (UNS). Teléfono: (54) (291) (4595144). Bahía Blanca, Argentina. 12 de Octubre 1198. fferrelli@criba.edu.ar. * Autor para correspondencia

2 Instituto Argentino de Oceanografía (IADO), Universidad Nacional del Sur (UNS)-CONICET, Bahía Blanca-Argentina. Teléfono: (54) (291) (4861112). B8000BFW, Bahía Blanca, Argentina. Florida 8000 (Camino La Carrindanga km 7,5) Complejo CCT CONICET Bahía Blanca, Edificio El.

Departamento de Geografía y Turismo, Universidad Nacional del Sur (UNS). Teléfono: (54) (291) (4595144). Bahía Blanca, Argentina. 12 de Octubre 1198. lujan.bustos@uns.edu.ar

3 Instituto Argentino de Oceanografía (IADO), Universidad Nacional del Sur (UNS)-CONICET, Bahía Blanca-Argentina. Teléfono: (54) (291) (4861112). B8000BFW, Bahía Blanca, Argentina. Florida 8000 (Camino La Carrindanga km 7,5) Complejo CCT CONICET Bahía Blanca, Edificio El.

Departamento de Geología, Universidad Nacional del Sur (UNS), 8000, Bahía Blanca, Argentina. Alem 1253, Cuerpo 2. gmeperillo@ criba.edu.ar

4 Instituto Argentino de Oceanografía (IADO), Universidad Nacional del Sur (UNS)-CONICET, Bahía Blanca-Argentina. Teléfono: (54) (291) (4861112). B8000BFW, Bahía Blanca, Argentina. Florida 8000 (Camino La Carrindanga km 7,5) Complejo CCT CONICET Bahía Blanca, Edificio El.

Departamento de Geografía y Turismo, Universidad Nacional del Sur (UNS). Teléfono: (54) (291) (4595144). Bahía Blanca, Argentina. 12 de Octubre 1198. ofpiccol@criba.edu.ar 


\begin{abstract}
An understanding of the climate of coastal cities is crucial for orientating urban land management. For this reason, it is essential to have access to long series of meteorological information with periods greater than 30 years. A lack of information or inconsistency can make climate study difficult. Numerical models are a suitable tool for the generation of climate data. For these reasons, this study aims to assess the precision and accuracy of temperature, relative humidity, and precipitation obtained in situ and derived from the Reanalysis NCEP/NCAR in eight coastal cities in different climates of Argentina. To do so, we studied the climatic series for the 1960-2019 period and those generated with the Reanalysis. Both series were statistically compared, considering diverse time scales and applying statistical methods. Time series were analysed using the quantile regression method to assess the study of the extremes in all the variables. The temperature presented a good agreement in climatic (60 years), monthly, and model scales. Relative humidity had higher results on the seasonal scale (mainly in winter and autumn), except for the cities located in warm and humid climates. Finally, rainfall registered good agreement in all temporal scales studied for cities located in temperate and arid climates. Results represent an essential database to orientate management plans and improve the quality of life and the coastal environment.
\end{abstract}

Keywords: Reanalysis NCEP/NCAR; in situ data; coastal cities; Argentina.

\title{
1. Introducción
}

En América Latina y el Caribe, la distribución espacial de la población destaca por el elevado nivel de urbanización. En los últimos 68 años la población urbana se incrementó del 45 al 80\% (CEPAL, 2018). Este rápido crecimiento urbano, sumado al cambio climático, afectaron varios aspectos de la vida humana, como la demanda de energía, la salud y la economía, en especial cuando no se tiene una buena planificación urbana (Romero, 2019; Takane, Kikegawa, Hara y Grimmond, 2019). Las ciudades costeras son especialmente sensibles al cambio climático por los riesgos directos en el aumento del nivel medio del mar, la ocurrencia de eventos extremos, etc. Por ello, el análisis del clima de las ciudades costeras es un elemento esencial al momento de realizar planificaciones urbanas. El conocimiento de la variabilidad climática depende directamente de la calidad de la información meteorológica de la que se dispone a escala local y regional (Ferrelli, Bustos, Piccolo, Huamantinco Cisneros y Perillo, 2016). En ciertos espacios, la escasez o inconsistencia de la información meteorológica genera dificultades para desarrollar investigaciones climáticas. Por tal motivo, en la actualidad, se desarrollaron estudios orientados a validar herramientas indirectas como modelos numéricos, datos de aeronaves, imágenes satelitales, etc. (Kalnay et al., 1996; Kistler et al., 2001; Bustos, Ferrelli y Piccolo, 2017; Devers, Vidal, Lauvernet, Graff y Vannier, 2020).

A escala regional, la distribución equidistante de estaciones meteorológicas que proporcionen información por períodos de tiempo superiores a 30 años es esencial para el estudio de la variabilidad climática (Bustos et al., 2017). Un modelo numérico que facilita la obtención de información mensual de distintas variables meteorológicas es el Reanalysis NCEP-NCAR. Este se encuentra validado a escala global y favorece la obtención de información de forma mensual desde el año 1948 hasta el presente (Kalnay et al., 1996; Kistler et al., 2001). Sin embargo, al ampliar la escala de análisis espacial, los datos del Reanalysis no se ajustan a las observaciones meteorológicas (Sachindra, Ahmed, Mamunur Rashid, Sehgal, Shahid y Perera, 2019; Nacar, Kankal y Okkan, 2019). Los datos del NCEP/NCAR necesitan ser corregidos al utilizarlos en escalas locales o regionales especialmente donde se presentan rasgos diferenciales como, por ejemplo, la topografía, tipo de ambiente, cercanía al mar, etc. (Seth, Rauscher, Camargo, Qian y Pal, 2007; Zhao, Guo y Fu, 2008; Viggiano, Busetto, Cimini, Di Paola, Geraldi, Ranghetti, Ricciardelli y Romano, 2019).

En Argentina, se ha utilizado este modelo numérico para completar series climáticas, analizar la variabilidad de las precipitaciones y estudiar eventos térmicos (Rusticucci y Kousky, 2002; Collazo, Barrucand y Rusticucci, 2019; Romero, González, Rolla y Losano, 2020). Sin embargo, no existe un estudio comparativo de lo que ocurre en ciudades costeras marinas y fluviales localizadas en distintas latitudes del país. En este contexto, tampoco se evidencia la existencia de una investigación que considere el ajuste del Reanalysis NCEP/NCAR a distintos tipos de clima de Argentina. La particularidad de estos espacios urbanos es que ofrecen una compleja gama de interacciones dinámicas y están, en general, sometidas a procesos de erosión, inundaciones y anegamientos (Piccolo, Merlotto, Bustos, Huamantinco Cisneros y García, 2017). En ellos, se generan procesos físicos, geológicos y biológicos que están en constante transformación y su estudio depende en gran medida del comportamiento variable del clima (Bustos et al., 
2017). Con la finalidad de obtener información válida para realizar estudios climáticos en áreas con escasez de datos, o con información faltante el objetivo de este estudio fue evaluar la precisión de los datos de temperatura del aire, humedad relativa y precipitación adquiridos del Reanalysis NCEP-NCAR para la caracterización climática a distintas escalas espacio-temporales de ciudades en zonas costeras marinas y fluviales de Argentina localizadas en distintas franjas climáticas. Cabe destacar que esos parámetros fueron seleccionados por ser los más utilizados en estimaciones climáticas.

\section{Metodología}

\section{1. Área de estudio}

El área de estudio está conformada por ciudades localizadas en costas marinas y fluviales pertenecientes a distintas regiones climáticas del país (Figura 1). Siguiendo los criterios de clasificación climática establecidos por Köppen, se estudiaron: i) Iguazú: (Misiones) representa las situaciones de una ciudad costera fluvial de clima subtropical sin estación seca, ii) Concordia (Entre Ríos) corresponde a una ciudad costera fluvial de clima templado pampeano, iii) Ciudad Autónoma de Buenos Aires: es la ciudad costera marino-fluvial más extensa de Argentina, localizada en clima templado húmedo, iv) Mar del Plata (Buenos Aires), al igual que en el caso anterior es una ciudad de clima templado húmedo, pero se localiza sobre las costas del Océano Atlántico por lo que la influencia oceánica es significativa, v) Bahía Blanca (Buenos Aires) es una ciudad costero-marina de clima templado de transición entre los climas húmedos y semiáridos, vi) Trelew (Chubut) es una ciudad costero-fluvial de clima templado según su temperatura y árido según se régimen pluviométrico, vii) Río Gallegos (Santa Cruz) localizada en la costa marina de clima frío; viii) Ushuaia (Tierra del Fuego) en los climas fríos húmedos de la cordillera y sobre la costa marina.

Figura 1. A. Clasificación climática de Köppen y B. Localización del área de estudio y estaciones meteorológicas
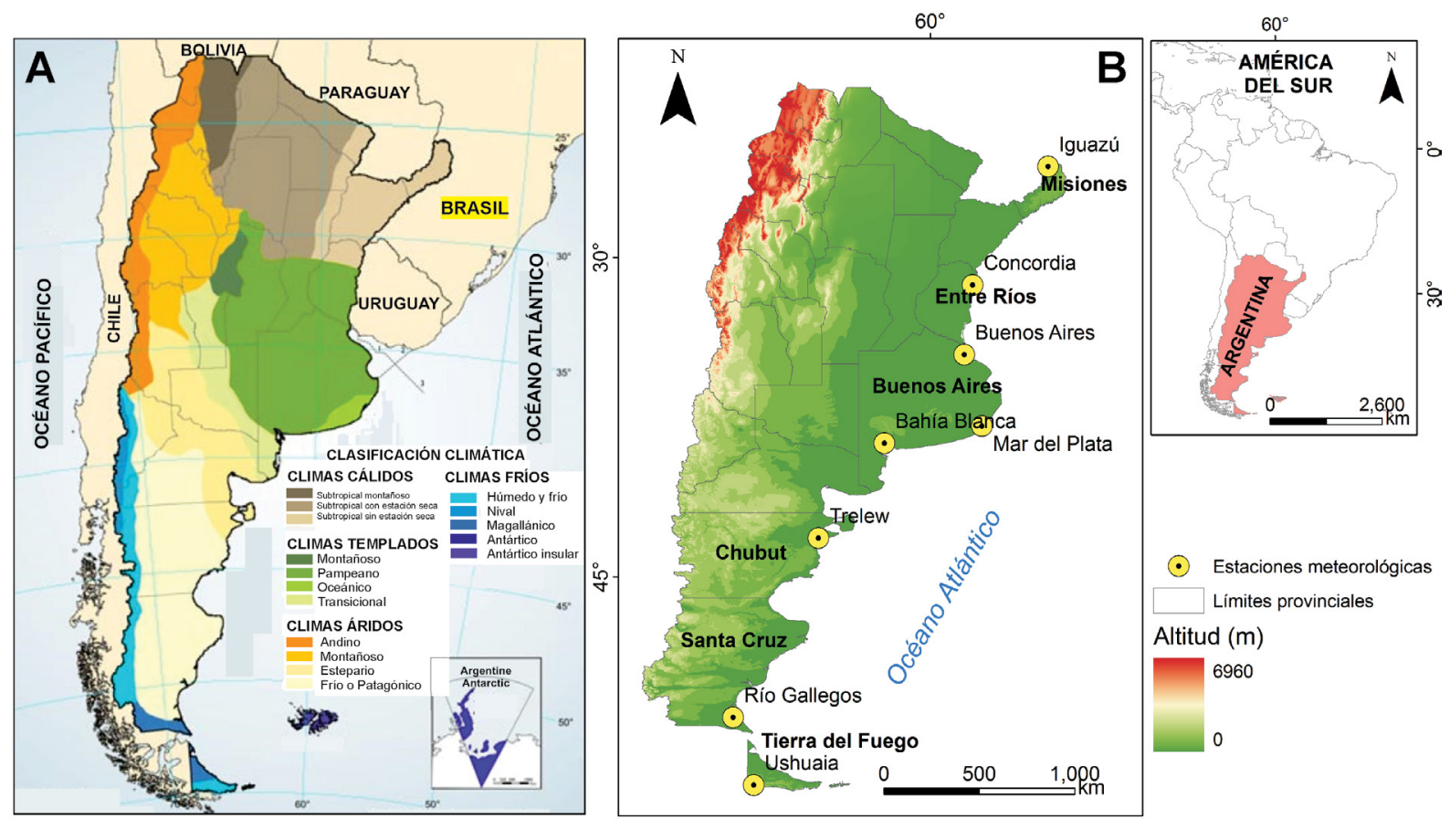

Elaboración propia

\subsection{Análisis estadísticos de las series de tiempo}

Se analizaron datos pertenecientes al Servicio Meteorológico Nacional (SMN, Argentina) y al Instituto Nacional de Tecnología Agropecuaria (INTA, Argentina). Se seleccionaron estaciones meteorológicas localizadas en ciudades costeras de Argentina (Figura 1). Los detalles de valores medios, localización absoluta, información perdida y valores atípicos se presentan en la Tabla 1. El período de estudio comprendió 1960-2019, exceptuando la estación de Ushuaia que tuvo información para el período 1991-2019. 
Se analizaron valores mensuales de temperatura del aire, humedad relativa y precipitación. Se trabajó con un total de 2.160 datos por estación, exceptuando Ushuaia, que tuvo 1.044 datos. En total, se analizaron 16.164 datos meteorológicos. Luego de que esta información fuese testeada, se sometió a pruebas de calidad y homogeneidad. En una primera instancia, los datos fueron ordenados en tablas para identificar posibles datos faltantes, inconsistencias y datos erróneos (Zhou, Aizen y Aizen, 2018). Para ello, se utilizaron los paquetes RclimDex (Zhang y Yang, 2013) y RHTest V4 (Wang, Wen y Wu, 2010) para el Software R. Esta información fue completada siguiendo los criterios descriptos en Ferrelli, Brendel, Aliaga, Piccolo y Perillo (2019). Se verificó que las ciudades estudiadas no excedieran el 5\% de información faltante e inconsistencias (Tabla 1).

Tabla 1. Estaciones meteorológicas utilizadas en este estudio

\begin{tabular}{|l|r|r|c|l|c|c|}
\hline Estaciones meteorológicas & Latitud & Longitud & Período de estudio & \multicolumn{1}{|c|}{ Provincia } & VA & I \\
\hline Iguazú & $-25,73$ & $-54,46$ & $1960-2019$ & Misiones & 0,94 & 0,66 \\
\hline Concordia & $-31,30$ & $-58,01$ & $1960-2019$ & Entre Ríos & 0,36 & 0,12 \\
\hline Buenos Aires & $-34,56$ & $-58,41$ & $1960-2019$ & Buenos Aires & 0,95 & 0,11 \\
\hline Mar del Plata & $-37,93$ & $-57,58$ & $1960-2019$ & Buenos Aires & 0,66 & 0,07 \\
\hline Bahía Blanca & $-38,73$ & $-62,17$ & $1960-2019$ & Buenos Aires & 0,25 & 0,06 \\
\hline Trelew & $-43,20$ & $-65,26$ & $1960-2019$ & Chubut & 0,87 & 0,25 \\
\hline Río Gallegos & $-51,61$ & $-69,28$ & $1960-2019$ & Santa Cruz & 0,88 & 0,12 \\
\hline Ushuaia & $-54,80$ & $-68,31$ & $1991-2019$ & Tierra del Fuego & 0,65 & 0,12 \\
\hline
\end{tabular}

VA. Valores atípicos e I. Inconsistencias. Ambos en valores porcentuales (\%)

Elaboración propia

Se descargaron datos de temperatura del aire, humedad relativa y precipitación del Reanalysis (NCEP/ NCAR) — de aquí en más Reanalysis- obtenidos a $1.000 \mathrm{mb}$, dado que está demostrado que los mismos son los más adecuados para realizar estudios climáticos (Ferrelli et al., 2016). Esta información fue brindada por el Centro de Predicción del Medio Ambiente Nacional (NCEP, Estados Unidos), el Centro Nacional de Investigación Atmosférica y la National Oceanic and Atmospheric Administration/Clima Diagnostic Center (NOAA/CDC, Estados Unidos). La ventaja que tiene este modelo es que genera información climática de libre acceso a una escala de $0,5^{\circ}$ de latitud por $0,5^{\circ}$ de longitud en su sitio web https://psl.noaa.gov. En este estudio, se recopilaron datos para cada una de las cuadrículas en las que se localizaron las ciudades costeras.

Las diferencias entre ambas fuentes de datos es que una representa información de sitio (estación meteorológica) y la otra un área (Reanalysis). Los análisis de esta información, se realizaron en distintas escalas espacio-temporales. Con la finalidad de observar y calcular las diferencias entre ambas fuentes de datos, se analizó la escala climática que hace referencia a todo el período (60 años, exceptuando Ushuaia que fue de 29 años) y las series de datos a escala anual, interanual, estacional y mensual. Se consideró el comportamiento espacio-temporal de tres series de datos: i. observadas: datos registrados por las estaciones meteorológicas, ii. Reanalysis: datos del modelo numérico sin modificar y iii. Modelados: el resultado de modelización estadística producto de la comparación de las dos series anteriores.

Para estudiar la validez de los datos obtenidos con el Reanalysis y las mediciones in situ, se aplicaron distintas pruebas estadísticas. En una primera instancia, se calcularon los valores medios de todo el período y los estacionales de ambas fuentes de información y sus diferencias. Posteriormente, se utilizó la prueba de Fisher para analizar si estos valores medios fueron estadísticamente iguales, considerando un $\alpha<5 \%$. Luego, se calculó el índice de determinación $\left(\mathrm{R}^{2}\right)$, los índices de correlación de Pearson y Spearman y la Concordancia. Adicionalmente, se aplicaron análisis de regresión lineal, polinómica, exponencial y logarítmica para estudiar ambas fuentes de datos. Como resultado, se obtuvieron fórmulas que permitieron modelar las variables meteorológicas obtenidas del Reanalysis para que representen con mayor exactitud y precisión el comportamiento interanual de los datos observados in situ. Finalmente, se aplicó el error cuadrático medio (RMSE) para medir la exactitud entre las series de datos. 


\subsection{Análisis de las series de tiempo por cuantiles}

Finalmente, se estudiaron las series de tiempo con la aplicación de una regresión cuantílica. Este procedimiento permitió evaluar el ajuste de las series de tiempo en los cuantiles más lejanos al valor medio y la mediana, es decir, en aquellos eventos térmicos fríos y húmedos y en los muy lluviosos y los secos. Si bien todos ellos componen situaciones normales dentro de los climas analizados, este análisis permitió identificar el ajuste del Reanalysis ante los distintos cuantiles.

Para ello, se realizaron regresiones cuantílicas dado que ha sido un método ampliamente utilizado para el estudio de las variables meteorológicas, principalmente para la precipitación (Cannon, Sobie y Murdock, 2015; Eum y Cannon, 2017).

En una primera instancia, se especificaron los cuantiles a estudiar considerando un valor de 0,1 para cada uno de ellos. De esta forma, el menor cuantil estudiado fue de 0,1 y el mayor de 0,9 , estableciendo un total de 10 cuantiles para las series de tiempo. Finalmente, para evaluar la precisión y exactitud de este análisis, se aplicaron el coeficiente de determinación $\mathrm{R}^{2}$, la intersección y el Error Medio Absoluto (MAE). Este procedimiento se llevó a cabo en el Software SPSS.

\section{Resultados}

\subsection{Análisis climático y estacional}

Del análisis comparativo de las series in situ con las del Reanalysis, se obtuvieron los valores medios con sus diferencias para la temperatura del aire, la humedad relativa y las precipitaciones que se presentan en la Tabla 2. Se identificó en las ciudades costero fluviales de clima cálido (Iguazú y Concordia) un patrón térmico más elevado que fue sobreestimado por el Reanalysis $\left(1,6\right.$ y $2,3^{\circ} \mathrm{C}$, respectivamente). La precipitación presentó la misma característica en ambas ciudades, superando los $400 \mathrm{~mm}$ en Concordia. Sin embargo, la humedad relativa tuvo valores similares con diferencias inferiores al $8 \%$ que están dentro del error de medición (Tabla 2).

En las ciudades que se localizan sobre la franja de climas templados (Buenos Aires, Mar del Plata y Bahía Blanca), las diferencias térmicas de ambas fuentes fueron menores a las analizadas anteriormente $\left(0,2,-0,9\right.$ y $-1,4^{\circ} \mathrm{C}$, respectivamente). La humedad relativa tuvo una diferencia menor al $6 \%$ en Mar del Plata, $8 \%$ en Bahía Blanca y solo de 0,8\% en Buenos Aires. En estas ciudades, la precipitación estuvo subestimada por el Reanalysis, alcanzando totales de precipitación de 293 y $240 \mathrm{~mm}$ menos que la observada. Asimismo, en Bahía Blanca, la precipitación solo presentó una diferencia de 14 mm (Tabla 2).

Las ciudades localizadas en latitudes altas tuvieron comportamientos diferentes. Por un lado Trelew y Ushuaia presentaron diferencias inferiores a $0,3^{\circ} \mathrm{C}$ en la temperatura del aire y de $5 \%$ en la humedad relativa. En el caso de Trelew, la precipitación también tuvo un valor similar en ambas series de datos (5 mm de diferencia), mientras que en Ushuaia la discrepancia fue mayor $(55,1 \mathrm{~mm})$. Rio Gallegos tuvo mayores diferencias entre ambas fuentes de información en todos los parámetros meteorológicos analizados (Tabla 2).

Tabla 2. Valores medios para el período 1960-2019 y sus diferencias según los datos observados in situ y del Reanalysis

\begin{tabular}{|l|r|r|r|r|r|r|r|r|r|}
\hline E.M. & \multicolumn{1}{c|}{ To } & \multicolumn{1}{c|}{ TR } & \multicolumn{1}{c|}{ To-TR } & \multicolumn{1}{c|}{ HRo } & \multicolumn{1}{c|}{ HRR } & HRo-HRR & \multicolumn{1}{l|}{ PPo } & \multicolumn{1}{c|}{ PPR } & PPo-PPR \\
\hline Iguazú & 21,1 & 22,7 & $-1,6$ & 80,6 & 86,1 & $-5,5$ & 1.855 & $2.017,1$ & $-162,3$ \\
\hline Concordia & 18,8 & 21,1 & $-2,3$ & 72,3 & 79,7 & $-7,4$ & 1.353 & $1.799,7$ & $-446,3$ \\
\hline Buenos Aires & 16,5 & 16,3 & 0,2 & 73,9 & 74,7 & $-0,8$ & 997,1 & 703,5 & 293,6 \\
\hline Mar del Plata & 13,9 & 14,8 & $-0,9$ & 79,3 & 73,5 & 5,8 & 924,1 & 684,1 & 240 \\
\hline Bahía Blanca & 15,3 & 16,7 & $-1,4$ & 64,1 & 56,4 & 7,7 & 644,4 & 630,1 & 14,3 \\
\hline Trelew & 13,6 & 13,8 & $-0,2$ & 52,4 & 56,2 & $-3,8$ & 199,9 & 194,9 & 5 \\
\hline Río Gallegos & 7,8 & 6,6 & 1,2 & 65,1 & 86,9 & $-21,8$ & 251,5 & 590,1 & $-338,6$ \\
\hline Ushuaia* & 5,9 & 6 & $-0,1$ & 74,9 & 79,5 & $-4,6$ & 513,7 & 458,6 & 55,1 \\
\hline
\end{tabular}

To. Temperatura observada, TR. Temperatura del Reanalysis, HRo. Humedad relativa observada, HRR, humedad relativa del Reanalysis, PPo, precipitación observada y PPR, precipitación del Reanalysis. * La estación de Ushuaia corresponde al período $1990-2019$ 
Por otro lado, se estudiaron los comportamientos de la temperatura del aire, la humedad relativa y las precipitaciones a escala estacional. Se observó que en el verano, se registraron las menores diferencias entre las temperaturas in situ (To) y las adquiridas del Reanalysis (TR). Estas fueron inferiores a $1^{\circ} \mathrm{C}$ en todas las ciudades costeras, excepto en Concordia $\left(2^{\circ} \mathrm{C}\right)$ y Río Gallegos $\left(3,2^{\circ} \mathrm{C}\right)$. En el otoño y el invierno, las ciudades localizadas al sur presentaron diferencias inferiores a los $0,8^{\circ} \mathrm{C}$. En contraposición, las ciudades localizadas más al norte tuvieron valores superiores a $1{ }^{\circ} \mathrm{C}$, exceptuando Buenos Aires $\left(0,1\right.$ y $0,5^{\circ} \mathrm{C}$ para cada estación, respectivamente). Finalmente, la primavera fue la estación del año que mayores diferencias térmicas presentó al analizar ambas fuentes de datos (Tabla 3).

La humedad relativa presentó dos patrones. Por un lado, las ciudades localizadas en los climas templados (Buenos Aires, Mar del Plata y Bahía Blanca) presentaron diferencias inferiores al $10 \%$ en todas las estaciones, excepto en la primavera. Este comportamiento también se apreció en Trelew y Ushuaia. Por otro lado, las ciudades de clima subtropical y frío tuvieron diferencias amplias, llegando a superar el $20 \%$. De igual manera, en todas las ciudades las diferencias mínimas se registraron en el invierno, excepto Bahía Blanca y Trelew (Tabla 3).

Tabla 3. Valores medios estacionales para el período 1960-2019 y sus diferencias según los datos observados in situ y los del Reanalysis

\begin{tabular}{|c|c|c|c|c|c|c|c|c|c|c|c|c|}
\hline & \multicolumn{3}{|c|}{ Verano } & \multicolumn{3}{|c|}{ Otoño } & \multicolumn{3}{|c|}{ Invierno } & \multicolumn{3}{|c|}{ Primavera } \\
\hline & To & $\mathrm{TR}$ & To-TR & To & TR & To-TR & To & TR & To-TR & To & TR & To-TR \\
\hline Iguazú & 25,3 & 25,6 & $-0,3$ & 20,7 & 22,2 & $-1,5$ & 16,3 & 19 & $-2,7$ & 21,3 & 23,9 & $-2,6$ \\
\hline Concordia & 24,8 & 26,8 & -2 & 19 & 20,8 & $-1,8$ & 13 & 15,2 & $-2,2$ & 18,7 & 20,1 & $-1,4$ \\
\hline Buenos Aires & 22,7 & 21,9 & 0,8 & 16,7 & 16,8 & $-0,1$ & 10,3 & 10,8 & $-0,5$ & 16,2 & 15,6 & 0,6 \\
\hline Mar del Plata & 19,5 & 19,9 & $-0,4$ & 14,7 & 15,7 & -1 & 8,4 & 9,9 & $-1,5$ & 13,1 & 13,6 & $-0,5$ \\
\hline Bahía Blanca & 22,5 & 23,5 & -1 & 15,1 & 16,4 & $-1,3$ & 8,4 & 9,9 & $-1,5$ & 15,1 & 16,4 & $-1,3$ \\
\hline Trelew & 20,4 & 19,8 & 0,6 & 13,6 & 13,8 & $-0,2$ & 6,7 & 7,5 & $-0,8$ & 13,8 & 13,2 & 0,6 \\
\hline Río Gallegos & 13 & 9,8 & 3,2 & 7,5 & 7,1 & 0,4 & 1,9 & 2,7 & $-0,8$ & 8,3 & 5,9 & 2,4 \\
\hline \multirow[t]{3}{*}{ Ushuaia* } & 9,2 & 8,4 & 0,8 & 6,1 & 6,2 & $-0,1$ & 2,2 & 2,7 & $-0,5$ & 5,9 & 5,2 & 0,7 \\
\hline & \multicolumn{3}{|c|}{ Verano } & \multicolumn{3}{|c|}{ Otoño } & \multicolumn{3}{|c|}{ Invierno } & \multicolumn{3}{|c|}{ Primavera } \\
\hline & HRo & HRR & HRo-HRR & HRo & HRR & HRo-HRR & HRo & HRR & HRo-HRR & HRo & HRR & HRo-HRR \\
\hline Iguazú & 78,2 & 90,1 & $-11,9$ & 83,4 & 90,3 & $-6,9$ & 83,7 & 83 & 0,7 & 76,7 & 80,8 & $-4,1$ \\
\hline Concordia & 65,4 & 80,7 & $-15,3$ & 76,2 & 81,4 & $-5,2$ & 79,1 & 78,8 & 0,3 & 69,6 & 78 & $-8,4$ \\
\hline Buenos Aires & 67,5 & 64,4 & 3,1 & 77,6 & 74,7 & 2,9 & 78,7 & 77,8 & 0,9 & 71,9 & 68,1 & 3,8 \\
\hline Mar del Plata & 75,5 & 70,1 & 5,4 & 80,8 & 75,3 & 5,5 & 82,2 & 76,4 & 5,8 & 78,5 & 70,1 & 8,4 \\
\hline Bahía Blanca & 53,8 & 45,9 & 7,9 & 68,8 & 69,9 & $-1,1$ & 71,2 & 62,3 & 8,9 & 61,2 & 50,9 & 10,3 \\
\hline Trelew & 42,4 & 45,5 & $-3,1$ & 55,9 & 60,3 & $-4,4$ & 63,6 & 69,4 & $-5,8$ & 48 & 52,8 & $-4,8$ \\
\hline Río Gallegos & 54,4 & 83 & $-28,6$ & 68,5 & 88,9 & $-20,4$ & 78,1 & 92,1 & -14 & 59,9 & 85,2 & $-25,3$ \\
\hline \multirow[t]{3}{*}{ Ushuaia* } & 71,9 & 77,6 & $-5,7$ & 77,3 & 83,1 & $-5,8$ & 79,2 & 87,1 & $-7,9$ & 71,1 & 79,1 & -8 \\
\hline & \multicolumn{3}{|c|}{ Verano } & \multicolumn{3}{|c|}{ Otoño } & \multicolumn{3}{|c|}{ Invierno } & \multicolumn{3}{|c|}{ Primavera } \\
\hline & PPo & PPR & PPo-PPR & PPo & PPR & PPo-PPR & PPo & PPR & PPo-PPR & PPo & PPR & PPo-PPR \\
\hline Iguazú & 495,1 & 720,1 & -225 & 453,1 & 464,4 & $-11,3$ & 365,4 & 260,4 & 105 & 541,3 & 521,9 & 19,4 \\
\hline Concordia & 385,7 & 444,9 & $-59,2$ & 395,7 & 445,5 & $-49,8$ & 212,2 & 569,5 & -357 & 359,7 & 439,2 & $-79,5$ \\
\hline Buenos Aires & 296,7 & 202,9 & 93,8 & 272,7 & 186,1 & 86,6 & 164,8 & 126,3 & 38,5 & 262,9 & 186,1 & 76,8 \\
\hline Mar del Plata & 275,8 & 243,7 & 32,1 & 249,4 & 162,2 & 87,2 & 177,3 & 114,5 & 62,8 & 221,7 & 166,5 & 55,2 \\
\hline Bahía Blanca & 200,9 & 229,9 & -29 & 155,4 & 154,1 & 1,3 & 101,6 & 95,4 & 6,2 & 188,6 & 183,3 & 5,3 \\
\hline Trelew & 46,7 & 54,9 & $-8,2$ & 61,3 & 55,2 & 6,1 & 48,2 & 58,7 & $-10,5$ & 43,6 & 53,3 & $-9,7$ \\
\hline Río Gallegos & 82,3 & 260,1 & -178 & 66,8 & 136,6 & $-69,8$ & 53,1 & 91,3 & $-38,2$ & 49,2 & 138,6 & $-89,4$ \\
\hline Ushuaia* & 137,6 & 227,2 & $-89,6$ & 143,2 & 142,9 & 0,3 & 125 & 108,3 & 16,7 & 107,9 & 132,1 & $-24,2$ \\
\hline
\end{tabular}

To. Temperatura observada, TR. Temperatura del Reanalysis, HRo. Humedad relativa observada, HRR, humedad relativa del Reanalysis, PPo, precipitación observada y PPR, precipitación del Reanalysis. * La estación de Ushuaia corresponde al período 1990-2019

Elaboración propia

La precipitación presentó amplias diferencias estacionales entre los datos observados (PPo) y los obtenidos del Reanalysis (PPR) en todas las ciudades. Sin embargo, se identificaron dos situaciones (Bahía 
Blanca y Trelew) en la que los valores medios tuvieron diferencias inferiores a $10 \mathrm{~mm}$ por estación. Los resultados más adecuados se obtuvieron en el invierno y el otoño de Trelew, la primavera de Bahía Blanca y en el otoño de Ushuaia. A pesar de ello, las precipitaciones estivales fueron las que mayores diferencias presentaron (Tabla 3).

\subsection{Análisis anual}

Se evaluaron los ajustes de las series observadas con respecto a las del Reanálisis para obtener una nueva serie derivada de un análisis de regresión (datos modelados). Esta nueva serie fue testeada nuevamente con los datos in situ. Para ello, se consideraron los datos anuales para el período de estudio (Tabla 4).

Sin embargo, la temperatura del aire de Buenos Aires, Mar del Plata, Trelew y Ushuaia y la precipitación y temperatura de Iguazú presentaron buenos resultados cuando las series fueron corregidas mediante un análisis de regresión (Tabla 4). Se observó que al aplicar las modelizaciones estadísticas, las series modeladas se asemejaron a las observadas y existió evidencia de que ambas fueron estadísticamente similares, considerando un $\alpha<0,05$ (Figura 2).

Tabla 4. Análisis estadísticos aplicados sobre las series observadas in situ respecto a las obtenidas con el Reanalysis (SO) y las modeladas resultantes del análisis de regresión (SM) para el período 1960-2019

\begin{tabular}{|c|c|c|c|c|c|c|c|c|c|c|c|}
\hline & & \multicolumn{2}{|c|}{ Fisher } & \multicolumn{2}{|c|}{$\mathrm{R}^{2}$} & \multicolumn{2}{|c|}{ Pearson } & \multicolumn{2}{|c|}{ Spearman } & \multicolumn{2}{|c|}{ Concordancia } \\
\hline & & $\mathrm{SO}$ & SM & SO & SM & SO & SM & SO & SM & SO & SM \\
\hline \multirow{3}{*}{ Iguazú } & $\mathrm{T}$ & 0,29 & 0,01 & 0,27 & 0,51 & 0,34 & 0,56 & 0,47 & 0,61 & 0,06 & 0,36 \\
\hline & HR & 0,89 & 0,25 & 0,03 & 0,33 & 0,15 & 0,29 & 0,22 & 0,36 & 0,02 & 0,18 \\
\hline & PP & 0,28 & 0,01 & 0,28 & 0,52 & 0,35 & 0,58 & 0,31 & 0,59 & 0,11 & 0,38 \\
\hline \multirow{3}{*}{ Concordia } & $\mathrm{T}$ & 0,84 & 0,69 & 0,04 & 0,11 & 0,08 & 0,09 & 0,11 & 0,11 & 0,01 & 0,01 \\
\hline & HR & 0,91 & 0,56 & 0,01 & 0,05 & 0,01 & 0,05 & 0,01 & 0,06 & 0,01 & 0,01 \\
\hline & PP & 0,99 & 0,85 & 0,01 & 0,01 & 0,01 & 0,01 & 0,01 & 0,01 & 0,01 & 0,01 \\
\hline \multirow{3}{*}{ Buenos Aires } & $\mathrm{T}$ & 0,22 & 0,02 & 0,41 & 0,69 & 0,55 & 0,71 & 0,58 & 0,72 & 0,41 & 0,66 \\
\hline & HR & 0,51 & 0,12 & 0,21 & 0,35 & 0,28 & 0,39 & 0,33 & 0,45 & 0,15 & 0,26 \\
\hline & PP & 0,74 & 0,11 & 0,26 & 0,35 & 0,35 & 0,41 & 0,39 & 0,45 & 0,25 & 0,36 \\
\hline \multirow{3}{*}{ Mar del Plata } & $\mathrm{T}$ & 0,12 & 0,01 & 0,61 & 0,89 & 0,62 & 0,88 & 0,68 & 0,91 & 0,59 & 0,75 \\
\hline & $\mathrm{HR}$ & 0,86 & 0,52 & 0,03 & 0,27 & 0,11 & 0,36 & 0,16 & 0,41 & 0,09 & 0,24 \\
\hline & PP & 0,91 & 0,41 & 0,08 & 0,39 & 0,16 & 0,41 & 0,21 & 0,46 & 0,04 & 0,31 \\
\hline \multirow{3}{*}{ Bahía Blanca } & $\mathrm{T}$ & 0,99 & 0,56 & 0,01 & 0,16 & 0,01 & 0,22 & 0,01 & 0,26 & 0,01 & 0,18 \\
\hline & HR & 0,99 & 0,54 & 0,01 & 0,05 & 0,01 & 0,01 & 0,01 & 0,01 & 0,01 & 0,01 \\
\hline & PP & 0,89 & 0,69 & 0,02 & 0,18 & 0,01 & 0,19 & 0,01 & 0,21 & 0,01 & 0,12 \\
\hline \multirow{3}{*}{ Trelew } & $\mathrm{T}$ & 0,21 & 0,03 & 0,54 & 0,84 & 0,61 & 0,86 & 0,58 & 0,89 & 0,49 & 0,78 \\
\hline & $\mathrm{HR}$ & 0,35 & 0,12 & 0,14 & 0,38 & 0,21 & 0,48 & 0,23 & 0,51 & 0,18 & 0,36 \\
\hline & PP & 0,29 & 0,14 & 0,28 & 0,36 & 0,35 & 0,46 & 0,39 & 0,51 & 0,22 & 0,41 \\
\hline \multirow{3}{*}{ Río Gallegos } & $\mathrm{T}$ & 0,59 & 0,21 & 0,13 & 0,31 & 0,21 & 0,36 & 0,22 & 0,38 & 0,11 & 0,29 \\
\hline & HR & 0,99 & 0,84 & 0,01 & 0,01 & 0,01 & 0,01 & 0,01 & 0,01 & 0,01 & 0,01 \\
\hline & PP & 0,99 & 0,51 & 0,18 & 0,38 & 0,21 & 0,41 & 0,26 & 0,46 & 0,01 & 0,22 \\
\hline \multirow{3}{*}{ Ushuaia* } & $\mathrm{T}$ & 0,06 & 0,01 & 0,71 & 0,91 & 0,75 & 0,94 & 0,72 & 0,96 & 0,66 & 0,88 \\
\hline & HR & 0,72 & 0,34 & 0,16 & 0,33 & 0,21 & 0,36 & 0,26 & 0,42 & 0,17 & 0,33 \\
\hline & PP & 0,99 & 0,59 & 0,02 & 0,25 & 0,03 & 0,28 & 0,03 & 0,29 & 0,01 & 0,15 \\
\hline
\end{tabular}

*El período de estudio de la ciudad de Ushuaia fue de 1991-2019 
Figura 2. Distribución interanual de la temperatura del aire y la precipitación considerando datos observados, del Reanalysis y modelados con análisis de regresión. Gráficos correspondientes a las ciudades con mejor correlación de datos del área de estudio
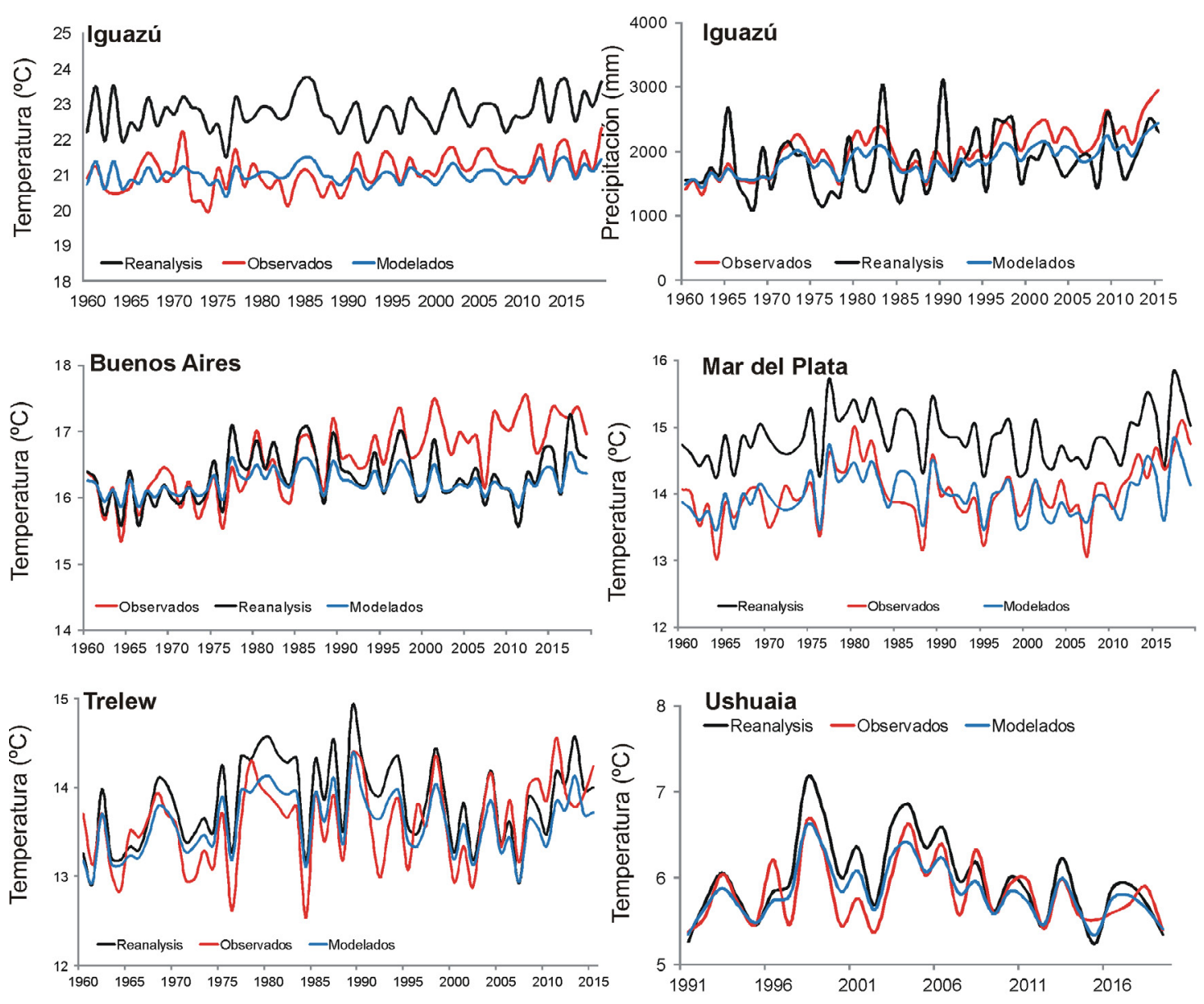

Elaboración propia

\subsection{Análisis mensual}

A partir de los estudios realizados, se procedió a analizar las variables meteorológicas, considerando los valores medios mensuales del período 1960-2019. Se observaron buenos resultados estadísticos $(\mathrm{p}<0,05)$ al analizar el comportamiento entre ambas fuentes de información. En general, los resultados máximos se obtuvieron al estudiar la temperatura del aire. Esta variable arrojó excelentes resultados en todas las ciudades costeras, con valores de $\mathrm{R}^{2}$ que oscilaron entre 0,94 y 0,99 , con índices de correlación superiores a 0,95 y con concordancia superior a 0,91 (Tabla 5).

Por otro lado, la humedad relativa no presentó ajustes estadísticamente significativos (valor $\mathrm{p}>0,05$ ) en las ciudades de Iguazú y Concordia que son de clima tropical y subtropical. Esta variable no pudo ser corregida estadísticamente con análisis de regresión. Sin embargo, en el resto de las ciudades, se obtuvieron resultados con valores de $\mathrm{R}^{2}$ superiores a 0,73 , índices de Pearson y Spearman superiores a 0,74 y concordancia superior a 0,71 (Tabla 5). La precipitación también presentó buenos ajustes $(\mathrm{p}<0,05)$, permitiendo generar una serie modelada a partir de los datos del Reanalysis que se adaptaron a los datos observados. El test de Fisher, identificó que los valores medios de las series transformadas presentaron el mismo valor medio que las adquiridas in situ $(\mathrm{p}<0,05)$ (Tabla 5).

Finalmente, en lo que respecta al error cuadrático medio (RSME), se evidenció que la temperatura presentó valores inferiores a 0,5 en todas las ciudades estudiadas, siendo menor en Río Gallegos $(0,21)$ y mayor en Concordia $(0,49)$. La humedad relativa tuvo valores que oscilaron entre 1,71 (Trelew) y 3,24 (Mar del Plata) y la precipitación fue la variable con mayores resultados de RSME, registrando 61,9 en Trelew y 324,1 en Iguazú (Tabla 5). 
Tabla 5. Análisis estadísticos realizados con las series observadas y modeladas de las ciudades del área de estudio

\begin{tabular}{|c|c|c|c|c|c|c|c|c|c|}
\hline & & Iguazú & Concordia & $\begin{array}{l}\text { Buenos } \\
\text { Aires }\end{array}$ & $\begin{array}{c}\text { Mar } \\
\text { del Plata }\end{array}$ & $\begin{array}{l}\text { Bahía } \\
\text { Blanca }\end{array}$ & Trelew & $\begin{array}{c}\text { Río } \\
\text { Gallegos }\end{array}$ & Ushuaia \\
\hline \multirow{3}{*}{ Fisher } & $T$ & 0,01 & 0,01 & 0,01 & 0,01 & 0,01 & 0,01 & 0,01 & 0,02 \\
\hline & HR & 0,25 & 0,85 & 0,02 & 0,05 & 0,04 & 0,01 & 0,01 & 0,04 \\
\hline & $\mathrm{PP}$ & 0,05 & 0,03 & 0,02 & 0,02 & 0,02 & 0,05 & 0,05 & 0,05 \\
\hline \multirow{3}{*}{$\mathrm{R} 2$} & $\mathrm{~T}$ & 0,94 & 0,98 & 0,99 & 0,99 & 0,99 & 0,99 & 0,94 & 0,97 \\
\hline & HR & 0,11 & 0,01 & 0,87 & 0,73 & 0,78 & 0,99 & 0,98 & 0,81 \\
\hline & $\mathrm{PP}$ & 0,65 & 0,81 & 0,94 & 0,91 & 0,91 & 0,69 & 0,73 & 0,75 \\
\hline \multirow{3}{*}{ Pearson } & $\mathrm{T}$ & 0,95 & 0,99 & 0,99 & 0,99 & 0,99 & 0,99 & 0,95 & 0,98 \\
\hline & HR & 0,15 & 0,01 & 0,85 & 0,74 & 0,81 & 0,99 & 0,96 & 0,82 \\
\hline & $\mathrm{PP}$ & 0,68 & 0,82 & 0,95 & 0,91 & 0,89 & 0,71 & 0,71 & 0,77 \\
\hline \multirow{3}{*}{ Spearman } & $\mathrm{T}$ & 0,96 & 0,99 & 0,99 & 0,99 & 0,99 & 0,99 & 0,95 & 0,97 \\
\hline & HR & 0,16 & 0,01 & 0,86 & 0,77 & 0,81 & 0,99 & 0,95 & 0,83 \\
\hline & $\mathrm{PP}$ & 0,71 & 0,82 & 0,96 & 0,92 & 0,93 & 0,73 & 0,71 & 0,77 \\
\hline \multirow{3}{*}{ Concordancia } & $\mathrm{T}$ & 0,91 & 0,97 & 0,99 & 0,98 & 0,99 & 0,99 & 0,91 & 0,94 \\
\hline & HR & 0,11 & 0,01 & 0,82 & 0,71 & 0,75 & 0,99 & 0,92 & 0,78 \\
\hline & $\mathrm{PP}$ & 0,69 & 0,77 & 0,92 & 0,89 & 0,89 & 0,72 & 0,72 & 0,71 \\
\hline \multirow{3}{*}{ RSME } & $\mathrm{T}$ & 0,43 & 0,49 & 0,35 & 0,28 & 0,33 & 0,31 & 0,21 & 0,29 \\
\hline & HR & 2,41 & 3,21 & 2,17 & 3,24 & 2,12 & 1,71 & 2,14 & 2,32 \\
\hline & PP & 324,1 & 298,6 & 191,2 & 181 & 154 & 61,9 & 98,5 & 86,5 \\
\hline
\end{tabular}

T. Temperatura, HR. Humedad Relativa, PP. Precipitación. RSME. Error Cuadrático Medio

Elaboración propia

Posteriormente, se aplicó un análisis de regresión (lineal, polinómica, logarítmica y exponencial) para la generación de fórmulas para la modelización estadística. De esta manera, se ajustaron los datos del Reanalysis a los observados en el terreno. En la tabla 6 , se presentan las fórmulas que permitieron estimar con precisión y ajuste estadístico cada uno de los parámetros climáticos. Como se mencionó anteriormente, en el caso de Iguazú y Concordia, no se identificó un ajuste adecuado para la humedad relativa, por lo que no fue posible su modelización. Esto se debió principalmente a que las series obtenidas con el Reanalysis sobreestimaron el parámetro durante todo el año. La temperatura del aire presentó ajustes lineales, mientras que la humedad relativa y la precipitación presentaron, además, ajustes polinómicos (Tabla 6). En la Tabla 6 se presentaron las fórmulas para ajustar las series in situ con las del Reanalysis que presentaron un valor $\mathrm{p}<0,05$.

Tabla 6. Fórmulas para la modelización climática de la temperatura del aire, la humedad relativa y la precipitación en ciudades costeras de Argentina

\begin{tabular}{|c|c|c|c|}
\hline & Temperatura & Humedad Relativa & Precipitación \\
\hline Iguazú & To $=$ TR $\times 1,2342-7,1947$ & Sin ajuste & $\mathrm{PPo}=0,0029 \times \mathrm{PPR}^{2}+1,2465 \times \mathrm{PPR}+41,815$ \\
\hline Concordia & $\mathrm{To}=\mathrm{TR} \times 0,8654+1,6565$ & Sin ajuste & $\mathrm{PPo}=0,0019 \times \mathrm{PPR}^{2}-1,0817 \times \mathrm{PPR}+246,82$ \\
\hline Buenos Aires & To $=$ TR $\times 1,1139-1,6777$ & $\begin{array}{c}\text { HRo }=0,0272 \times \mathrm{HRR}^{2}+4,8248 \mathrm{HRR} \\
-131,98\end{array}$ & $\mathrm{PPo}=1,2748 \times \mathrm{PPR}+8,3599$ \\
\hline $\begin{array}{l}\text { Mar del } \\
\text { Plata }\end{array}$ & $\mathrm{To}=\mathrm{TR} \times 1,102-2,3752$ & $\mathrm{HRo}=0,7056 \times \mathrm{HRR}+27,426$ & PPo $=-0,0123 \times \mathrm{PPR}^{2}+2,1667 \times$ PPR $-1,204$ \\
\hline Bahía Blanca & To $=$ TR $\times 1,0311-1,9568$ & $\mathrm{HRo}=0,6819 \times \mathrm{HRR}+27,786$ & $\mathrm{PPo}=-0,0168 \times \mathrm{PPR}^{2}+2,4869 \mathrm{PPR}-24,364$ \\
\hline Trelew & To $=$ TR $\times 1,0977-1,6463$ & HRo $=0,9353 \times$ HRR $-0,0593$ & 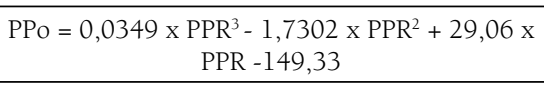 \\
\hline Río Gallegos & To $=$ TR $\times 1,5129-2,2577$ & HRo $=2,3858 \times$ HRR $-142,04$ & $\mathrm{PPo}=0,1896 \times \mathrm{PPR}+11,361$ \\
\hline Ushuaia & To $=$ TR $\times 1,2052-1,4006$ & HRo $=0,7394 \times \mathrm{HRR}+16,085$ & $\begin{array}{c}\mathrm{PPo}=-0,0034 \times \mathrm{PPR}^{3}+0,40233 \mathrm{PPR}^{2}-16,39 \\
\mathrm{PPR}+238,7\end{array}$ \\
\hline
\end{tabular}

To. Temperatura observada, TR. Temperatura del Reanalysis, HRo. Humedad Relativa observada, HRR. Humedad Relativa del Renalysis, PPo. Precipitación observada y PPR. Precipitación del Reanalysis 
Figura 3. Distribución de los datos medios mensuales de temperatura del aire del Reanalysis, los observados y los modelados para las ciudades costeras de Argentina
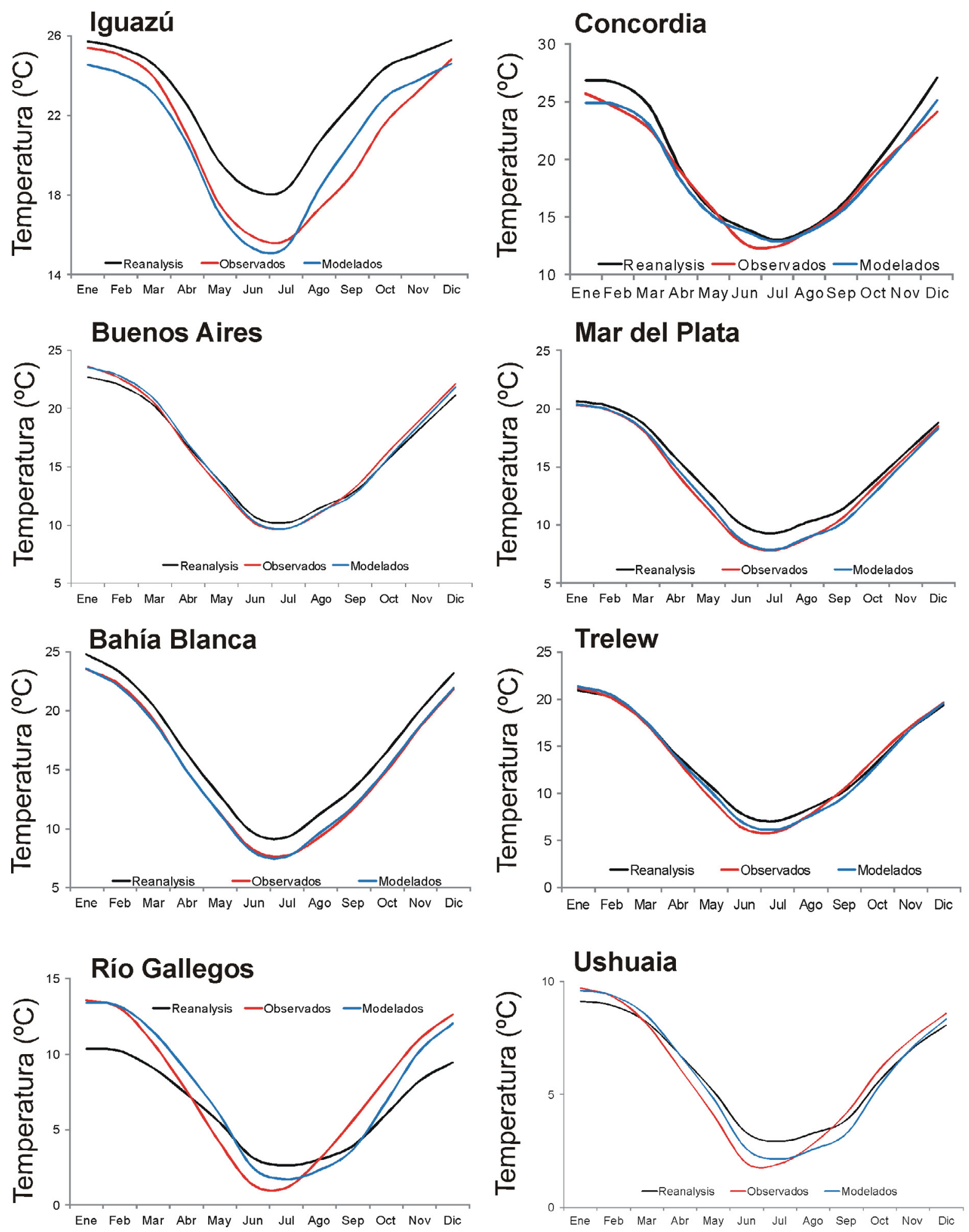

Elaboración propia

Como resultado de la evaluación estadística realizada, se obtuvo la distribución mensual de la temperatura del aire, la humedad relativa y la precipitación adquirida con datos observados, modelados y derivados 
del Reanalysis (Figuras 3, 4 y 5). La temperatura del aire presentó un patrón similar al obtenido in situ una vez que se modelaron las series del Reanalysis. En general, este último sobreestimó el comportamiento térmico. Sin embargo, la aplicación de las fórmulas presentadas en la tabla 6 generaron que las series modeladas representen adecuadamente los valores observados, con la evidencia estadística de estar representando el mismo valor medio, considerando los resultados de un test de Fisher con $\mathrm{p}<0,05$ (Figura 3).

En cuanto a la humedad relativa se observó que las series modeladas se comportaron de manera similar a las observadas, en especial en los climas fríos (Figura 4). En tanto, los datos del Reanalysis tuvieron un comportamiento mensual similar a los observados, pero en la mayoría de los casos sobreestimaron los registros mensuales (Figura 4). Finalmente, el ajuste de la precipitación fue complejo. Si bien existe evidencia de que los datos modelados presentaron una similitud con los medidos en el terreno, no se logra identificar con claridad las estaciones secas, como en el caso de Concordia (Figura 5). A pesar de ello, la modelación estadística permite comprender la distribución mensual de las precipitaciones en las ciudades costeras de Argentina (Figura 5).

Figura 4. Distribución de los datos medios mensuales de humedad relativa del Reanalysis, los observados y los modelados para las ciudades costeras de Argentina
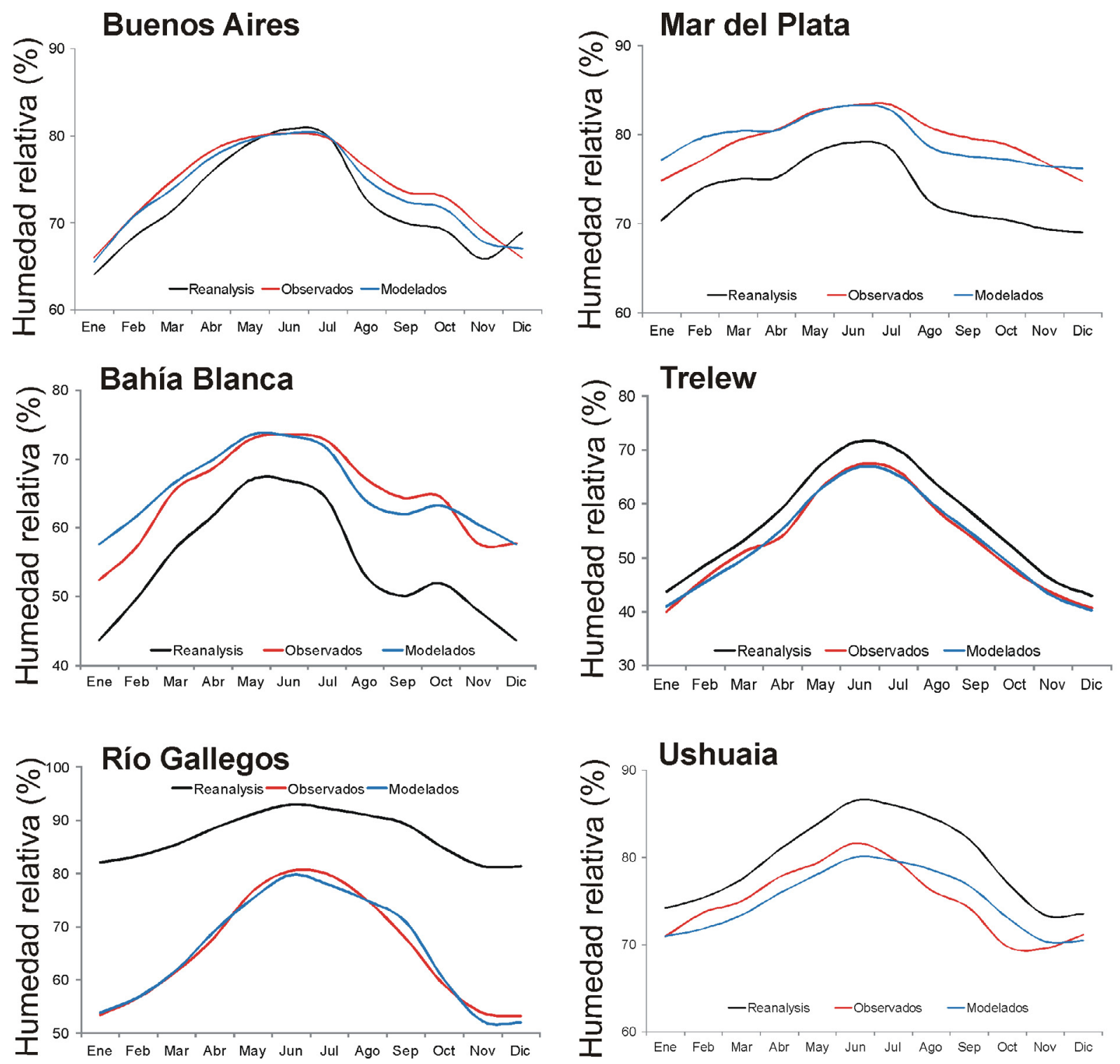

Elaboración propia 
Figura 5. Distribución de los datos medios mensuales de precipitación del Reanalysis, los observados y los modelados para las ciudades costeras de Argentina
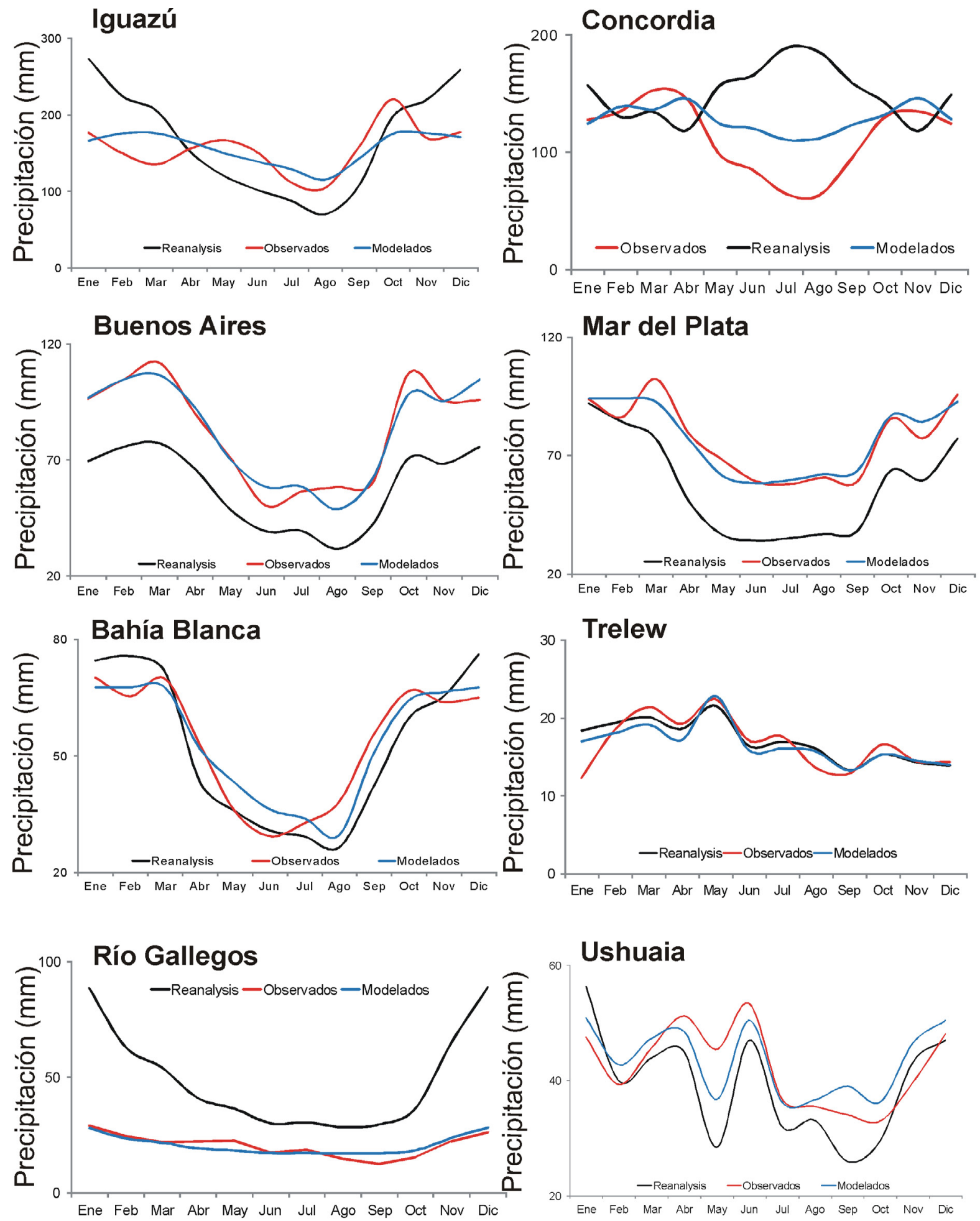

Elaboración propia

\subsection{Análisis del ajuste estadístico por cuantiles}

En este apartado, se detallaron los resultados obtenidos al analizar las series de tiempo observadas con aquellas obtenidas del Reanalysis a través de un análisis de regresión cuantílica. En lo que respecta a 
la temperatura, se observó que en la ciudad de Iguazú (clima subtropical sin estación seca) los cuantiles (de aquí en más q) mayores (q8 y q9) no presentaron resultados estadísticamente significativos (p>0,05). Una situación similar de identificó en Concordia, donde los máximos y los mínimos q no tuvieron significancia estadística considerando un valor $\mathrm{p}<0,05$.

Las ciudades localizadas en climas templados (Buenos Aires y Mar del Plata) tuvieron significancia en la mayor parte de los q $(p<0,05)$, exceptuando el q9 de Buenos Aires $(p>0,05)$. Trelew tuvo valores significativos en todos los $\mathrm{q}$, al igual que Ushuaia $(\mathrm{p}<0,05)$. Por su parte, Río Gallegos no tuvo resultados significativos al analizar q1 q2 y q3 ( $p>0,05)$ (Tabla 7$)$.

Tabla 7. Resultados de la regresión cuantílica obtenidos para los valores de temperatura de las ciudades costeras estudiadas

\begin{tabular}{|c|c|c|c|c|c|c|c|c|c|c|}
\hline \multirow{4}{*}{ Iguazú } & Q & $\mathrm{q}=0,1$ & $\mathrm{q}=0,2$ & $\mathrm{q}=0,3$ & $\mathrm{q}=0,4$ & $\mathrm{q}=0,5$ & $q=0,6$ & $\mathrm{q}=0,7$ & $\mathrm{q}=0,8$ & $\mathrm{q}=0,9$ \\
\hline & MAE & 0,6 & 0,4 & 0,4 & 0,3 & 0,3 & 0,3 & 0,4 & 0,4 & 0,7 \\
\hline & Int & 4,2 & 5,6 & 10,1 & 12,7 & 12,0 & 10,5 & 14,9 & 14,2 & 18,4 \\
\hline & $\mathrm{R}^{2}$ & 0,9 & 0,8 & 0,6 & 0,5 & 0,5 & 0,6 & 0,4 & 0,4 & 0,2 \\
\hline \multirow{4}{*}{ Concordia } & Q & $\mathrm{q}=0,1$ & $q=0,2$ & $q=0,3$ & $\mathrm{q}=0,4$ & $\mathrm{q}=0,5$ & $q=0,6$ & $\mathrm{q}=0,7$ & $\mathrm{q}=0,8$ & $\mathrm{q}=0,9$ \\
\hline & MAE & 0,7 & 0,5 & 0,4 & 0,4 & 0,4 & 0,4 & 0,4 & 0,5 & 0,7 \\
\hline & Int & 25,7 & 22,7 & 20,9 & 18,2 & 22,3 & 22,9 & 23,8 & 22,1 & 27,1 \\
\hline & $\mathrm{R}^{2}$ & 0,0 & 0,2 & 0,3 & 0,5 & 0,2 & 0,2 & 0,2 & 0,3 & 0,0 \\
\hline \multirow{4}{*}{ Buenos Aires } & Q & $\mathrm{q}=0,1$ & $q=0,2$ & $q=0,3$ & $\mathrm{q}=0,4$ & $\mathrm{q}=0,5$ & $q=0,6$ & $q=0,7$ & $\mathrm{q}=0,8$ & $\mathrm{q}=0,9$ \\
\hline & MAE & 0,4 & 0,3 & 0,3 & 0,2 & 0,2 & 0,3 & 0,3 & 0,3 & 0,4 \\
\hline & Int & 10,9 & 9,8 & 10,0 & 8,1 & 7,3 & 5,9 & 4,8 & 6,4 & 5,5 \\
\hline & $\mathrm{R}^{2}$ & 0,3 & 0,4 & 0,4 & 0,5 & 0,5 & 0,6 & 0,7 & 0,6 & 0,7 \\
\hline \multirow{4}{*}{ Mar del Plata } & $\mathrm{Q}$ & $q=0,1$ & $\mathrm{q}=0,2$ & $q=0,3$ & $\mathrm{q}=0,4$ & $\mathrm{q}=0,5$ & $\mathrm{q}=0,6$ & $\mathrm{q}=0,7$ & $\mathrm{q}=0,8$ & $\mathrm{q}=0,9$ \\
\hline & MAE & 0,3 & 0,2 & 0,2 & 0,2 & 0,2 & 0,2 & 0,2 & 0,2 & 0,3 \\
\hline & Int & 2,9 & 4,5 & 5,0 & 5,7 & 5,4 & 5,4 & 4,8 & 5,8 & 6,0 \\
\hline & $\mathrm{R}^{2}$ & 0,8 & 0,7 & 0,7 & 0,7 & 0,7 & 0,7 & 0,7 & 0,7 & 0,7 \\
\hline \multirow{4}{*}{ Bahía Blanca } & $\mathrm{Q}$ & $\mathrm{q}=0,1$ & $\mathrm{q}=0,2$ & $\mathrm{q}=0,3$ & $q=0,4$ & $\mathrm{q}=0,5$ & $\mathrm{q}=0,6$ & $\mathrm{q}=0,7$ & $\mathrm{q}=0,8$ & $\mathrm{q}=0,9$ \\
\hline & MAE & 0,7 & 0,6 & 0,5 & 0,5 & 0,4 & 0,5 & 0,5 & 0,6 & 0,8 \\
\hline & Int & 13,0 & 12,8 & 16,1 & 17,4 & 16,6 & 19,3 & 17,8 & 20,6 & 17,8 \\
\hline & $\mathrm{R}^{2}$ & 0,2 & 0,2 & 0,0 & 0,1 & 0,0 & 0,2 & 0,5 & 0,2 & 0,0 \\
\hline \multirow{4}{*}{ Trelew } & $\mathrm{Q}$ & $\mathrm{q}=0,1$ & $\mathrm{q}=0,2$ & $q=0,3$ & $\mathrm{q}=0,4$ & $\mathrm{q}=0,5$ & $q=0,6$ & $\mathrm{q}=0,7$ & $\mathrm{q}=0,8$ & $\mathrm{q}=0,9$ \\
\hline & MAE & 0,5 & 0,4 & 0,3 & 0,3 & 0,3 & 0,3 & 0,3 & 0,4 & 0,4 \\
\hline & Int & 2,4 & 5,3 & 5,7 & 3,9 & 4,2 & 4,7 & 2,8 & 1,8 & 1,1 \\
\hline & $\mathrm{R}^{2}$ & 0,8 & 0,6 & 0,6 & 0,7 & 0,7 & 0,7 & 0,8 & 0,9 & 1,0 \\
\hline \multirow{4}{*}{ Río Gallegos } & $\mathrm{Q}$ & $\mathrm{q}=0,1$ & $q=0,2$ & $\mathrm{q}=0,3$ & $q=0,4$ & $q=0,5$ & $q=0,6$ & $q=0,7$ & $\mathrm{q}=0,8$ & $\mathrm{q}=0,9$ \\
\hline & MAE & 0,5 & 0,4 & 0,3 & 0,3 & 0,3 & 0,3 & 0,3 & 0,4 & 0,5 \\
\hline & Int & 5,5 & 5,3 & 5,7 & 4,9 & 4,5 & 4,1 & 4,1 & 3,7 & 3,0 \\
\hline & $\mathbf{R}^{2}$ & 0,1 & 0,1 & 0,1 & 0,2 & 0,3 & 0,3 & 0,3 & 0,4 & 0,5 \\
\hline \multirow{4}{*}{ Ushuaia } & $\mathrm{Q}$ & $\mathrm{q}=0,1$ & $\mathrm{q}=0,2$ & $\mathrm{q}=0,3$ & $\mathrm{q}=0,4$ & $\mathrm{q}=0,5$ & $q=0,6$ & $\mathrm{q}=0,7$ & $\mathrm{q}=0,8$ & $\mathrm{q}=0,9$ \\
\hline & MAE & 0,2 & 0,2 & 0,1 & 0,1 & 0,1 & 0,1 & 0,2 & 0,2 & 0,2 \\
\hline & Int & 2,1 & 2,1 & 1,0 & 1,1 & 1,3 & 1,0 & 1,4 & 1,8 & 2,3 \\
\hline & $\mathbf{R}^{2}$ & 0,6 & 0,6 & 0,8 & 0,8 & 0,8 & 0,8 & 0,8 & 0,7 & 0,6 \\
\hline
\end{tabular}

Q. Cuantiles, MAE. Error Medio Absoluto, Int. Intersección, R2. Coeficiente de determinación. Los cuantiles (q) señalados en negrita corresponden a los resultados estadísticamente significativos $(\mathrm{p}<0,05)$

Elaboración propia

Se observó que en las ciudades en las que se evidenció menor significancia, las pendientes de las líneas predictivas fueron muy variantes de acuerdo al número de q. Esta situación es claramente observable en Bahía Blanca. Sin embargo, en otras, como por ejemplo Ushuaia e Iguazú la pendiente fue positiva y similar en todos los q (Figura 6). 
Figura 6. Parámetros estimados para los distintos cuantiles, aquellos obtenidos por una regresión lineal y las líneas predictivas $(\mathrm{q}=1$ hasta $\mathrm{q}=9$ ) resultante del análisis de los datos de temperatura $(\mathrm{T})$ observada y obtenida del Reanalysis para las ciudades de Iguazú, Buenos Aires, Bahía Blanca y Ushuaia

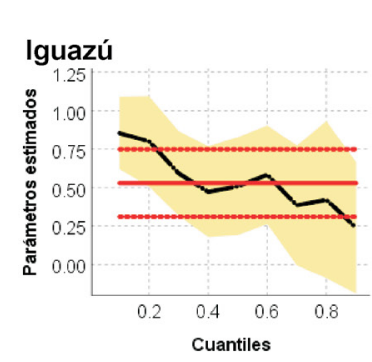

Buenos Aires

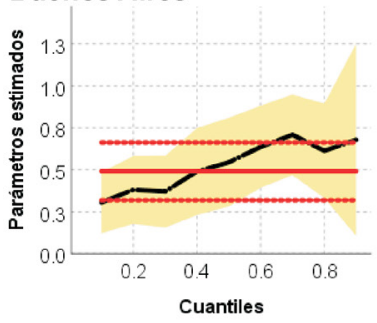

Cuantiles
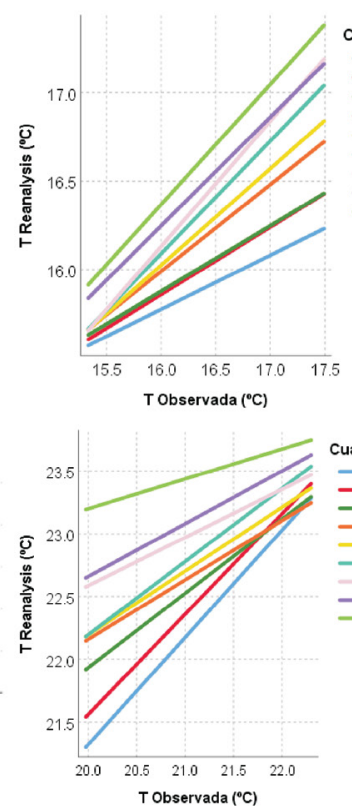
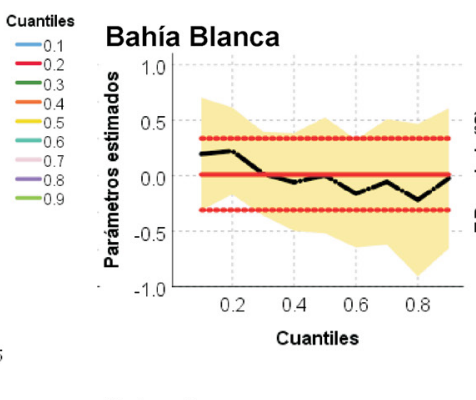

\section{Ushuaia}
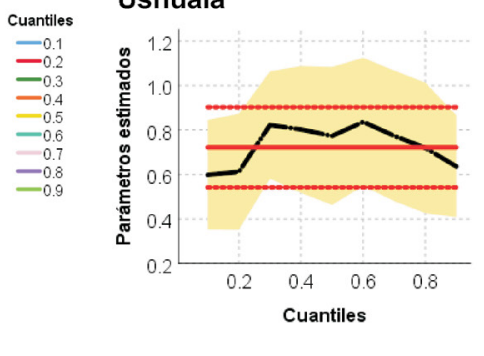
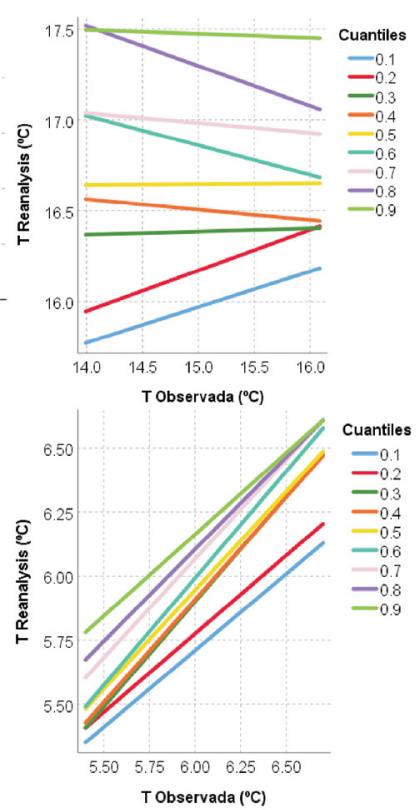

Cuantiles

Intervalos de confianza para los parámetros estimados (95\%) - Parámetros estimados para cada cuantil

- Parámetros estimados por una regresión lineal

... Intervalos de confianza para la regresión lineal (95\%)

Elaboración propia

$\mathrm{Al}$ analizar la humedad relativa por cuantiles, se estableció en general una baja significancia en los resultados obtenidos ( $p>0,05)$. Estos se evidenciaron en Buenos Aires (de q4 a q9), Mar del Plata (de q1 a q4), Bahía Blanca (de q2 a q5), Trelew (q1 a q5) y Ushuaia (q9) (Tabla 8). Esto pone de manifiesto que los datos obtenidos del Reanalysis para el estudio de la humedad relativa en las ciudades costeras estudiadas no permiten generar un buen ajuste entre los datos, tal y como se evidenció en el apartado 3.2.

Tabla 8. Resultados de la regresión cuantílica obtenidos para los valores de humedad relativa de las ciudades costeras estudiadas

\begin{tabular}{|c|c|c|c|c|c|c|c|c|c|c|}
\hline \multirow{4}{*}{ Iguazú } & $\mathrm{Q}$ & $\mathrm{q}=0,1$ & $q=0,2$ & $\mathrm{q}=0,3$ & $\mathrm{q}=0,4$ & $q=0,5$ & $q=0,6$ & $\mathrm{q}=0,7$ & $\mathrm{q}=0,8$ & $\mathrm{q}=0,9$ \\
\hline & MAE & 3,8 & 2,6 & 2,2 & 2,1 & 1,9 & 2,0 & 2,2 & 2,5 & 3,3 \\
\hline & Int & 96,9 & 77,2 & 74,2 & 75,8 & 68,2 & 65,6 & 67,9 & 56,5 & 73,2 \\
\hline & $\mathrm{R}^{2}$ & $-0,2$ & 0,1 & 0,1 & 0,1 & 0,2 & 0,3 & 0,2 & 0,4 & 0,2 \\
\hline \multirow{4}{*}{ Concordia } & $\mathrm{Q}$ & $q=0,1$ & $q=0,2$ & $q=0,3$ & $q=0,4$ & $q=0,5$ & $q=0,6$ & $q=0,7$ & $q=0,8$ & $\mathrm{q}=0,9$ \\
\hline & MAE & 1,8 & 1,2 & 1,1 & 1,0 & 1,0 & 1,0 & 1,1 & 1,3 & 1,5 \\
\hline & Int & 72,9 & 81,3 & 84,2 & 88,2 & 84,8 & 85,8 & 89,5 & 93,7 & 91,5 \\
\hline & $\mathrm{R}^{2}$ & 0,1 & 0,0 & 0,1 & 0,1 & 0,1 & 0,1 & 0,1 & 0,2 & 0,1 \\
\hline \multirow{4}{*}{ Buenos Aires } & $\mathrm{Q}$ & $\mathrm{q}=0,1$ & $q=0,2$ & $\mathrm{q}=0,3$ & $\mathrm{q}=0,4$ & $q=0,5$ & $q=0,6$ & $\mathrm{q}=0,7$ & $q=0,8$ & $\mathrm{q}=0,9$ \\
\hline & MAE & 3,3 & 2,3 & 2,0 & 1,9 & 1,8 & 1,9 & 2,1 & 2,4 & 3,1 \\
\hline & Int & $-20,0$ & 18,3 & 28,6 & 27,7 & 38,1 & 30,9 & 34,1 & 43,4 & 48,6 \\
\hline & $\mathrm{R}^{2}$ & 1,2 & 0,7 & 0,6 & 0,6 & 0,5 & 0,6 & 0,5 & 0,4 & 0,4 \\
\hline \multirow{4}{*}{ Mar del Plata } & $\mathrm{Q}$ & $q=0,1$ & $q=0,2$ & $q=0,3$ & $q=0,4$ & $q=0,5$ & $q=0,6$ & $q=0,7$ & $\mathrm{q}=0,8$ & $\mathrm{q}=0,9$ \\
\hline & MAE & 3,0 & 2,1 & 1,9 & 1,8 & 1,6 & 1,7 & 1,8 & 2,1 & 2,9 \\
\hline & Int & 44,5 & 53,3 & 50,8 & 55,6 & 68,1 & 69,4 & 68,5 & 74,8 & 94,6 \\
\hline & $\mathrm{R}^{2}$ & 0,3 & 0,2 & 0,3 & 0,2 & 0,1 & 0,1 & 0,1 & 0,0 & $-0,2$ \\
\hline \multirow{4}{*}{ Bahía Blanca } & $\mathrm{Q}$ & $\mathrm{q}=0,1$ & $q=0,2$ & $\mathrm{q}=0,3$ & $q=0,4$ & $q=0,5$ & $q=0,6$ & $\mathrm{q}=0,7$ & $\mathrm{q}=0,8$ & $\mathrm{q}=0,9$ \\
\hline & MAE & 6,1 & 4,1 & 3,7 & 3,4 & 3,3 & 3,4 & 3,7 & 4,9 & 6,2 \\
\hline & Int & 53,6 & 32,5 & 37,1 & 40,6 & 45,1 & 58,4 & 57,5 & 76,9 & 67,5 \\
\hline & $\mathrm{R}^{2}$ & 0,1 & 0,3 & 0,2 & 0,2 & 0,1 & 0,0 & 0,0 & 0,3 & 0,1 \\
\hline
\end{tabular}




\begin{tabular}{|c|c|c|c|c|c|c|c|c|c|c|}
\hline \multirow{4}{*}{ Trelew } & $\mathrm{Q}$ & $q=0,1$ & $q=0,2$ & $q=0,3$ & $\mathrm{q}=0,4$ & $q=0,5$ & $q=0,6$ & $\mathrm{q}=0,7$ & $q=0,8$ & $q=0,9$ \\
\hline & MAE & 2,3 & 1,7 & 1,5 & 1,4 & 1,4 & 1,4 & 1,6 & 1,9 & 2,6 \\
\hline & Int & 42,1 & 43,7 & 41,0 & 43,5 & 44,9 & 48,8 & 47,0 & 51,5 & 50,6 \\
\hline & $\mathrm{R}^{2}$ & 0,2 & 0,2 & 0,3 & 0,2 & 0,2 & 0,2 & 0,2 & 0,1 & 0,2 \\
\hline \multirow{4}{*}{ Río Gallegos } & $\mathrm{Q}$ & $q=0,1$ & $q=0,2$ & $q=0,3$ & $q=0,4$ & $q=0,5$ & $q=0,6$ & $q=0,7$ & $\mathrm{q}=0,8$ & $q=0,9$ \\
\hline & MAE & 4,5 & 3,2 & 2,7 & 2,2 & 2,1 & 2,1 & 2,3 & 2,5 & 2,7 \\
\hline & Int & 70,0 & 86,0 & 95,8 & 84,8 & 81,8 & 80,0 & 80,7 & 83,9 & 81,9 \\
\hline & $\mathbf{R}^{2}$ & 0,2 & 0,0 & 0,2 & 0,0 & 0,1 & 0,1 & 0,1 & 0,1 & 0,1 \\
\hline \multirow{4}{*}{ Ushuaia } & $\mathrm{Q}$ & $\mathrm{q}=0,1$ & $\mathrm{q}=0,2$ & $q=0,3$ & $q=0,4$ & $q=0,5$ & $q=0,6$ & $\mathrm{q}=0,7$ & $\mathrm{q}=0,8$ & $\mathrm{q}=0,9$ \\
\hline & MAE & 4,0 & 2,7 & 2,3 & 2,3 & 2,2 & 2,4 & 2,5 & 3,2 & 3,6 \\
\hline & Int & 73,1 & 82,0 & 94,0 & 92,2 & 97,2 & 99,5 & 94,7 & 98,6 & 97,4 \\
\hline & $\mathrm{R}^{2}$ & 0,0 & 0,1 & 0,4 & 0,4 & 0,4 & 0,3 & 0,3 & 0,3 & 0,3 \\
\hline
\end{tabular}

Q. Cuantiles, MAE. Error Medio Absoluto, Int. Intersección, R2. Coeficiente de determinación. Los cuantiles (q) señalados en negrita corresponden a los resultados estadísticamente significativos $(\mathrm{p}<0,05)$

Elaboración propia

A modo de ejemplo, se presentan los casos de Concordia, Mar del Plata, Trelew y Río Gallegos donde se muestran los resultados obtenidos para los parámetros estimados para los q y aquellos obtenidos de una regresión lineal. Las líneas predictivas evidencian el comportamiento distinto que tienen las variables en cada uno de los quintiles. Las mayores diferencias se observaron en los extremos, dejando de manifiesto que el Reanalysis pierde precisión al estudiar eventos de humedad relativa elevada o muy baja (Figura 7).

Figura 7. Parámetros estimados para los distintos cuantiles, aquellos obtenidos por una regresión lineal y las líneas predictivas ( $\mathrm{q}=1$ hasta $\mathrm{q}=9$ ) resultante del análisis de los datos de humedad relativa (HR) observada y obtenida del Reanalysis para Concordia, Trelew, Mar del Plata y Río Gallegos
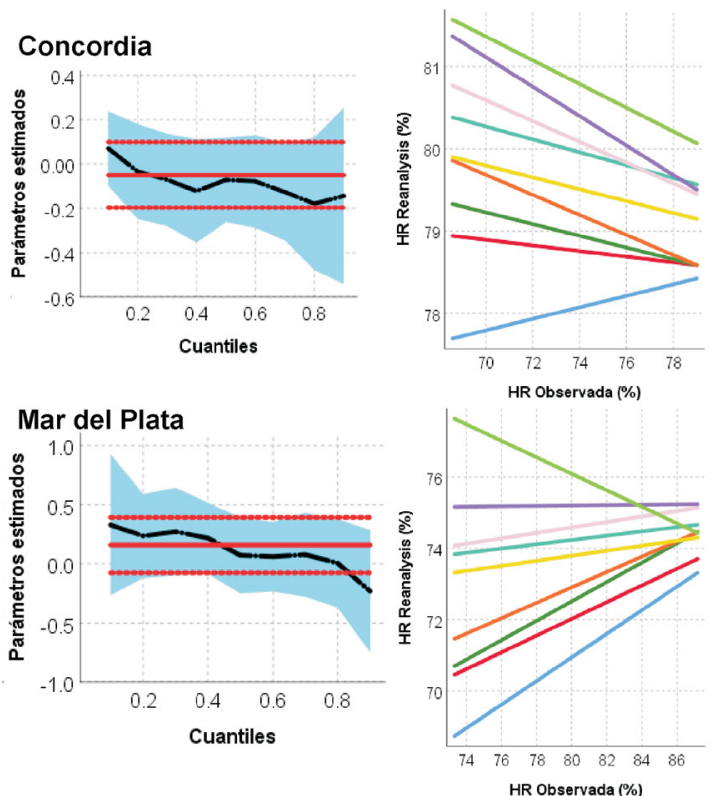

HR Observada (\%)

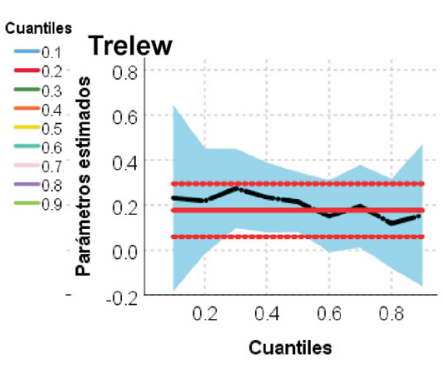

Río Gallegos

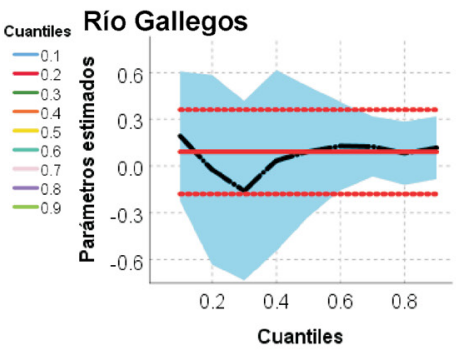

Cuantiles

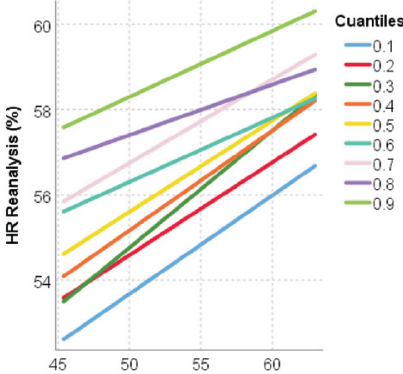

HR Observada (\%)

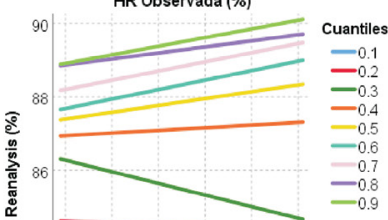

Intervalos de confianza para los parámetros estimados (95\%) — Parámetros estimados para cada cuantil

- Parámetros estimados por una regresión lineal

-ee- Intervalos de confianza para la regresión lineal $(95 \%)$

Elaboración propia

Finalmente, se realizó el estudio de la precipitación. Esta variable tuvo un comportamiento espacial heterogéneo según el tipo de clima que representó la ciudad costera estudiada. Por ejemplo, Iguazú tuvo resultados significativos en los 9 q analizados. Esta situación, se evidenció también en Trelew. Ambos coinciden con los climas donde las precipitaciones son más elevadas y más bajas, respectivamente (Tabla 9). 
Por otro lado, los cuantiles 6, 7 y 8 de Buenos Aires tuvieron significancia estadística y en Mar del Plata del q4 al q6. Una situación similar se presentó en Bahía Blanca. Esto dejó de manifiesto que los valores de precipitación fueron más precisos al analizar los cuantiles centrales de los distintos tipos de clima templados. El Reanalysis fue más impreciso a medida que se analizaron los eventos más lluviosos y los secos. Esta situación también se presentó en los climas fríos húmedos y nivales (Río Gallegos y Ushuaia, respectivamente) (Tabla 9).

Tabla 9. Resultados de la regresión cuantílica obtenidos para los valores de precipitación de las ciudades costeras estudiadas

\begin{tabular}{|c|c|c|c|c|c|c|c|c|c|c|}
\hline \multirow{4}{*}{ Iguazú } & Q & $\mathrm{q}=0,1$ & $q=0,2$ & $q=0,3$ & $\mathrm{q}=0,4$ & $q=0,5$ & $\mathrm{q}=0,6$ & $\mathrm{q}=0,7$ & $q=0,8$ & $q=0,9$ \\
\hline & MAE & 458,8 & 331,9 & 313,5 & 272,6 & 267,7 & 281,0 & 307,9 & 366,9 & 435,0 \\
\hline & Int & 1225,3 & 835,8 & 907,0 & 1318,7 & 1295,2 & 1287,2 & 1132,0 & 1499,8 & 1432,9 \\
\hline & $\mathrm{R}^{2}$ & 0,2 & 0,5 & 0,5 & 0,3 & 0,4 & 0,5 & 0,6 & 0,5 & 0,5 \\
\hline \multirow{4}{*}{ Concordia } & Q & $\mathrm{q}=0,1$ & $\mathrm{q}=0,2$ & $q=0,3$ & $q=0,4$ & $q=0,5$ & $\mathrm{q}=0,6$ & $\mathrm{q}=0,7$ & $\mathrm{q}=0,8$ & $\mathrm{q}=0,9$ \\
\hline & MAE & 666,5 & 527,1 & 399,6 & 361,6 & 350,8 & 355,6 & 389,0 & 457,2 & 572,0 \\
\hline & Int & 666,9 & 1271,0 & 1320,4 & 1695,7 & 2006,0 & 1988,6 & 2462,5 & 2307,0 & 2685,0 \\
\hline & $\mathbf{R}^{2}$ & 0,4 & 0,1 & 0,2 & 0,0 & $-0,1$ & 0,0 & $-0,3$ & $-0,1$ & $-0,2$ \\
\hline \multirow{4}{*}{ Buenos Aires } & Q & $\mathrm{q}=0,1$ & $q=0,2$ & $\mathrm{q}=0,3$ & $q=0,4$ & $q=0,5$ & $\mathrm{q}=0,6$ & $\mathrm{q}=0,7$ & $q=0,8$ & $q=0,9$ \\
\hline & MAE & 247,5 & 165,6 & 140,8 & 127,0 & 123,8 & 126,9 & 140,7 & 162,9 & 183,3 \\
\hline & Int & 144,0 & 143,9 & $-72,3$ & 99,9 & 207,6 & 274,7 & 307,4 & 312,2 & 307,6 \\
\hline & $\mathrm{R}^{2}$ & 0,3 & 0,4 & 0,7 & 0,6 & 0,5 & 0,5 & 0,5 & 0,5 & 0,6 \\
\hline \multirow{4}{*}{ Mar del Plata } & Q & $\mathrm{q}=0,1$ & $q=0,2$ & $\mathrm{q}=0,3$ & $q=0,4$ & $q=0,5$ & $\mathrm{q}=0,6$ & $\mathrm{q}=0,7$ & $\mathrm{q}=0,8$ & $\mathrm{q}=0,9$ \\
\hline & MAE & 229,6 & 188,8 & 142,2 & 136,5 & 133,1 & 137,7 & 146,9 & 169,2 & 254,5 \\
\hline & Int & 14,6 & 181,6 & 302,9 & 348,0 & 376,1 & 327,3 & 420,3 & 467,0 & 752,5 \\
\hline & $\mathrm{R}^{2}$ & 0,5 & 0,4 & 0,3 & 0,3 & 0,3 & 0,4 & 0,4 & 0,4 & 0,2 \\
\hline \multirow{4}{*}{ Bahía Blanca } & $\mathrm{Q}$ & $\mathrm{q}=0,1$ & $q=0,2$ & $\mathrm{q}=0,3$ & $\mathrm{q}=0,4$ & $\mathrm{q}=0,5$ & $\mathrm{q}=0,6$ & $\mathrm{q}=0,7$ & $\mathrm{q}=0,8$ & $\mathrm{q}=0,9$ \\
\hline & MAE & 240,5 & 199,7 & 169,7 & 164,2 & 159,0 & 164,6 & 172,2 & 246,8 & 360,1 \\
\hline & Int & 70,1 & 175,0 & 191,5 & 253,8 & 314,3 & 291,8 & 363,8 & 809,3 & 928,8 \\
\hline & $\mathrm{R}^{2}$ & 0,5 & 0,4 & 0,5 & 0,4 & 0,4 & 0,5 & 0,5 & 0,0 & 0,0 \\
\hline \multirow{4}{*}{ Trelew } & Q & $\mathrm{q}=0,1$ & $q=0,2$ & $q=0,3$ & $\mathrm{q}=0,4$ & $q=0,5$ & $\mathrm{q}=0,6$ & $\mathrm{q}=0,7$ & $q=0,8$ & $\mathrm{q}=0,9$ \\
\hline & MAE & 75,1 & 60,7 & 50,2 & 47,5 & 45,4 & 46,0 & 49,7 & 58,5 & 75,9 \\
\hline & Int & 47,0 & 74,4 & 80,7 & 98,0 & 119,4 & 136,4 & 134,5 & 108,2 & 133,5 \\
\hline & $\mathrm{R}^{2}$ & 0,4 & 0,4 & 0,4 & 0,4 & 0,4 & 0,3 & 0,4 & 0,6 & 0,6 \\
\hline \multirow{4}{*}{ Río Gallegos } & $\mathrm{Q}$ & $\mathrm{q}=0,1$ & $\mathrm{q}=0,2$ & $q=0,3$ & $\mathrm{q}=0,4$ & $\mathrm{q}=0,5$ & $\mathrm{q}=0,6$ & $\mathrm{q}=0,7$ & $\mathrm{q}=0,8$ & $\mathrm{q}=0,9$ \\
\hline & MAE & 212,4 & 168,7 & 129,5 & 116,7 & 114,5 & 118,5 & 137,8 & 159,1 & 221,1 \\
\hline & Int & 412,1 & 563,0 & 744,4 & 865,6 & 899,8 & 998,1 & 1060,4 & 1102,6 & 1058,6 \\
\hline & $\mathrm{R}^{2}$ & 0,1 & 0,5 & 0,9 & 0,2 & 0,3 & 0,5 & 0,5 & 0,5 & 0,8 \\
\hline \multirow{4}{*}{ Ushuaia } & $\mathrm{Q}$ & $\mathrm{q}=0,1$ & $q=0,2$ & $q=0,3$ & $q=0,4$ & $q=0,5$ & $\mathrm{q}=0,6$ & $\mathrm{q}=0,7$ & $\mathrm{q}=0,8$ & $\mathrm{q}=0,9$ \\
\hline & MAE & 4,0 & 2,7 & 2,3 & 2,3 & 2,2 & 2,4 & 2,5 & 3,2 & 3,6 \\
\hline & Int & 73,1 & 82,0 & 94,0 & 92,2 & 97,2 & 99,5 & 94,7 & 98,6 & 97,4 \\
\hline & $\mathbf{R}^{2}$ & 0,0 & 0,1 & 0,4 & 0,4 & 0,4 & 0,3 & 0,3 & 0,3 & 0,3 \\
\hline
\end{tabular}

Q. Cuantiles, MAE. Error Medio Absoluto, Int. Intersección, R2. Coeficiente de determinación. Los cuantiles (q) señalados en negrita corresponden a los resultados estadísticamente significaticos $(\mathrm{p}<0,05)$

Elaboración propia

Por último, se presentaron los ejemplos de los parámetros estimados por la regresión cuantílica, aquellos derivados de una regresión linear y las líneas predictivas para las ciudades de Iguazú, Mar del Plata, Trelew y Ushuaia. Se observó una correlación similar entre los q de Iguazú y Trelew, ambas ciudades con significancia estadística en todos los q. Por otro lado, Mar del Plata y Ushuaia presentaron líneas predictivas más inconsistentes (Figura 9). 
Figura 8. Parámetros estimados para los distintos cuantiles, aquellos obtenidos por una regresión lineal y las líneas predictivas ( $\mathrm{q}=1$ hasta $\mathrm{q}=9$ ) resultante del análisis de los datos de precipitación (PP) observada y obtenida del Reanalysis para las ciudades costeras estudiadas

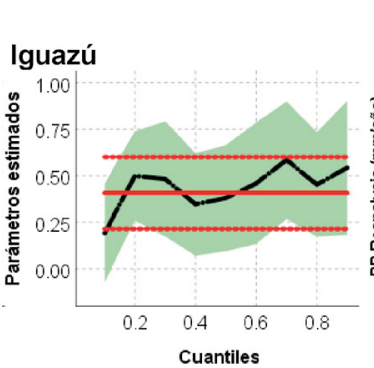

Mar del Plata

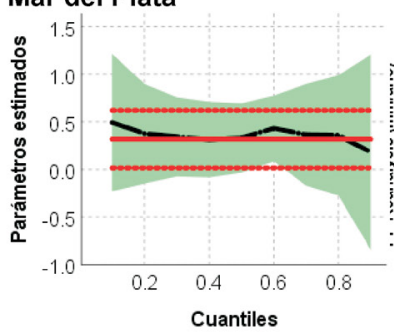

Cuantiles

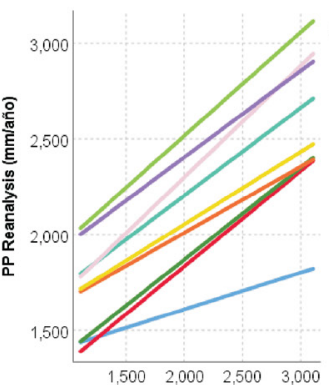

PP Observada (mmlaño)

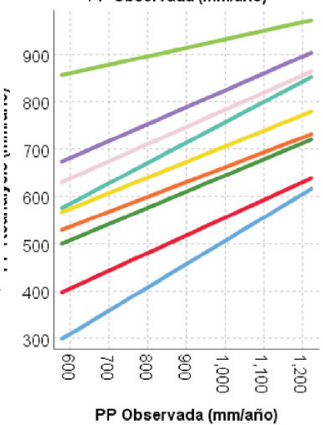

PP Observada (mm/año)

Intervalos de confianza para los parámetros estimados $(95 \%)$ - Parámetros estimados para cada cuanti

- Parámetros estimados por una regresión lineal

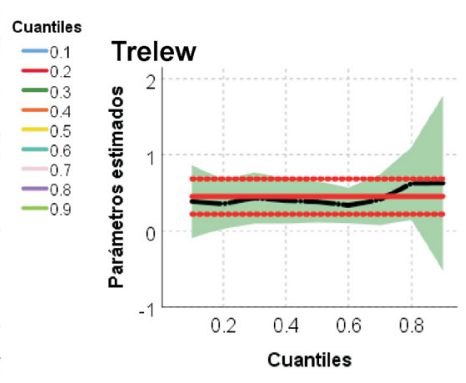

Ushuaia
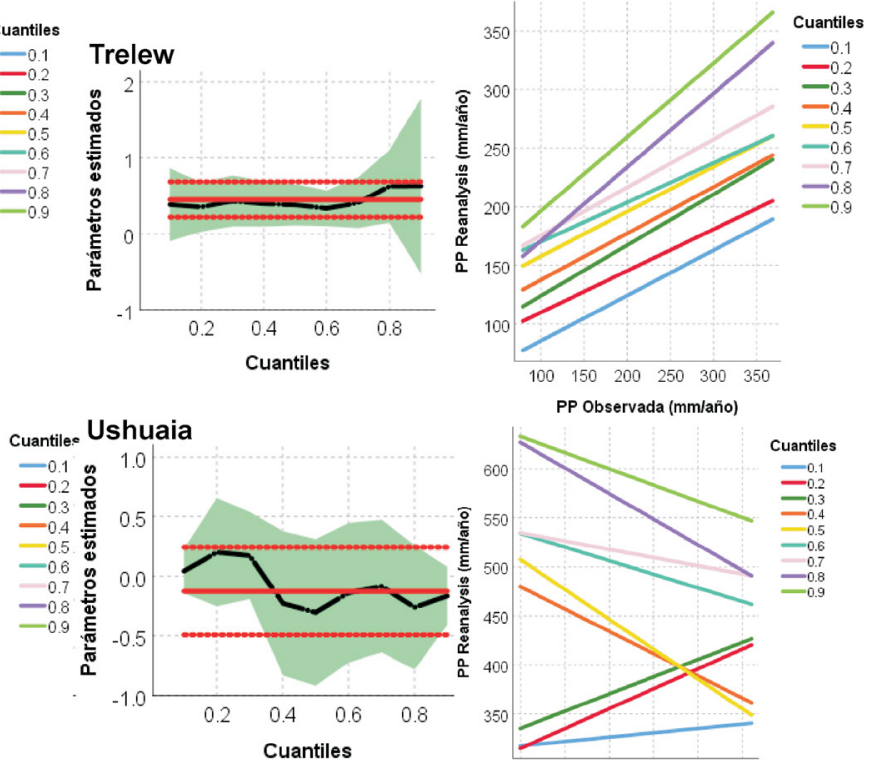

PP Observada (mm/año)

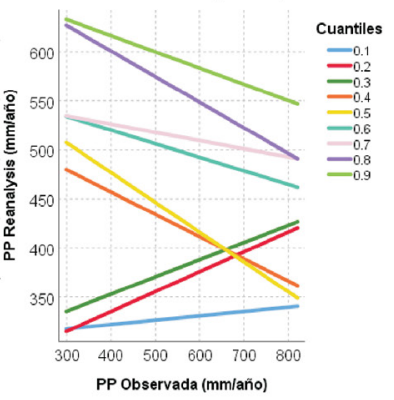

Elaboración propia

\section{Discusión de los resultados}

Se comprobó que el Reanalysis NCEP/NCAR es una fuente de datos indirecta que favorece la caracterización del clima de distintas ciudades costeras de Argentina. Disponer de una fuente de datos indirecta testeada para distintas regiones climáticas del país resulta de vital importancia para rellenar series incompletas y analizar la variabilidad climática (Libanda, Bwalya, Nkolola y Chilenkana, 2020). Los datos proporcionados por el Reanalysis han demostrado ser útiles en climas fríos, templados y cálidos de Sudamérica por su capacidad de identificar anomalías térmicas y barométricas que determinan el ingreso de frentes fríos y de esa forma utilizarlos, por ejemplo, en modelos de clima a futuro (Garreaud, 2000; Martins Andrade, Muller, Cavalcanti, Fernandez Long, Bidegain y Berri, 2012; da Rocha Repinaldo, Müller y Martins Andrade, 2017; Araya-Osses, Casanueva, Roman-Figueroa, Uribe y Paneque, 2020). Sin embargo, a distintas escalas espacio-temporales de análisis, se observaron particularidades que son necesarias considerar antes de aplicar este modelo. Por ejemplo, durante la primavera se presentaron diferencias notables que hacen que el estudio a escala estacional requiera de un análisis cuidadoso de la información. A pesar de ello, se destaca que el Reanalysis es uno de los modelos numéricos más utilizados a escala mundial debido al gran período de información disponible y a su resolución temporal (Mesinger et al., 2006, Viggiano et al., 2019). Sumado a ello, la mejor ventaja que tiene este modelo es que a partir de comparaciones y correcciones estadísticas se logra un downscaling de manera eficiente, permitiendo caracterizar los valores medios, el comportamiento interanual y mensual de las variables de temperatura del aire, humedad relativa y precipitación de manera adecuada (Zhao et al., 2008; Viggiano et al., 2019; Libanda et al., 2020). A pesar de ello, se destaca que en este estudio los valores extremos de temperatura, precipitación y/o humedad relativa no estuvieron bien estimados en todos los climas estudiados, dando evidencia de que es necesario realizar múltiples pruebas estadísticas para trabajar con las series de tiempo. A modo de ejemplo, se destaca que la precipitación se ajustó mejor en los climas subtropicales y áridos (Iguazú y Trelew) y en los cuantiles medios de los climas templados (Buenos Aires, Mar del Plata y Bahía Blanca).

En este contexto, es destacable que, en la literatura, existen numerosos métodos de downscaling. El análisis de regresión lineal, múltiple, exponencial, logarítmica y polinómica ha sido ampliamente 
utilizado (p.e., Ferrelli et al., 2016; Bustos et al., 2016). Sin embargo, otros métodos son importantes de ser incluidos para lograr una mejor precisión en los resultados finales. Los métodos de downscaling se aplican en todas las situaciones, aun cuando se observe que el modelo numérico representa de manera adecuada las condiciones climáticas de un determinado sitio (Heo, Anh, Shin y Kjeldser, 2019), especialmente en el estudio de las precipitaciones (Jeon, Paciorek y Wehner, 2016). Por esta razón, los modelos numéricos, como el Reanalysis deben ser corregidos a través de distintas técnicas de corrección (Boé, Terray, Habets y Martin, 2007; Abatzoglou y Brown, 2012; Hwang y Graham 2013). Dependiendo de las variables meteorológicas de interés existen distintos modelos que ayudan a mejorar la precisión de los datos obtenidos con el Reanalysis en relación a los observados in situ (Seo y Kim, 2018). Por ejemplo, en el estudio de las precipitaciones los métodos como el de mapeo por cuantiles y la regresión por cuantiles han resultado ser útiles para su estudio (Cannon et al., 2015; Eum y Cannon, 2017). Sin embargo, los métodos de regresión han presentado resultados considerables en el estudio de la temperatura y la humedad relativa. En nuestro trabajo, se evidenció que el ajuste lineal entre las variables de temperatura del aire demostró ser un buen estimador para corregir esta variable. La precipitación presentó ajustes más complejos y obtuvo resultados más adecuados cuando se aplicaron los métodos de regresión por cuantiles debido a que el mismo permite una mejor discriminación de lo que sucede en los extremos de las series analizadas (Cannon et al., 2015). Esto demuestra que la variabilidad propia de las precipitaciones es un elemento que no está bien representado por el Reanalysis, a pesar de que el mismo permita disponer de una amplia gama de datos. Finalmente, la humedad relativa ha presentado resultados altamente variables según los distintos climas de Argentina y eso se debe a la influencia de las masas de aire secas o humedad que afectan al territorio estudiado y originan cambios en el patrón hidrométrico.

El Reanalysis no podría ser utilizado para la caracterización climática de ciudades costeras de Argentina sin ser corregido adecuadamente, debido a que los errores producto de las diferencias de escala espacial, los generados por la topografía y la cercanía al mar, generan que la serie sea estadísticamente distinta a los datos observados (Kistler et al., 2001; Rusticucci y Kousky, 2002; Kim, Kim, Boo, Shim y Kim, 2019; Gomes de Lima y Rodrigues Alcântara, 2019). Es importante considerar que los métodos estadísticos aplicados para realizar un downscaling de variables meteorológicas permiten modelar las relaciones estacionarias entre una serie de datos observados a escala local con los provenientes del Reanalysis (Xue, Janjic, Dudhia, Nasicy y De Sales, 2014; Miró Pérez, Estrela Navarro y Olcina Cantos, 2015; Libanda et al., 2020). Con estos mecanismos, las series pueden ser reconstruidas y extrapoladas con los modelos estadísticos obtenidos para cada localidad (Coulibary, Dibike y Anctil, 2005; Miró Pérez, Estrela Navarro, Pastor y Millán Muñoz, 2009; Viggiano et al., 2019).

Por otro lado, la humedad relativa de las ciudades costeras localizadas en los climas subtropicales sin estación seca y los templados húmedos no pudieron ser corregidos a escala mensual e interanual. Esto se debe principalmente a que en algunas circunstancias, los datos del Reanalysis tienen diferencias espacio-temporales marcadas que impiden su modelización. Estas hacen referencia a los eventos extremos termo-pluviométricos y a las sobreestimaciones o subestimaciones de los valores en algunas estaciones del año (Haylock, Cawley, Harpham, Wilby y Goodess, 2006; Kim et al., 2019; Gomes de Lima y Rodrigues Alcântara, 2019). Por este motivo, la estación pluviométricamente seca de Concordia tampoco pudo ser determinada en el modelo identificado.

En cuanto a la comparación de las variables meteorológicas utilizadas en este estudio, se evidenció que en todos los casos, la temperatura del aire presentó los valores de correlación más elevados. Sin embargo, al analizarlos a escala estacional, los mismos tuvieron mayores diferencias con los datos observados. Esta situación fue demostrada también por otros autores (por ejemplo, Gomes de Lima y Rodrigues Alcântara, 2019). En lo que respecta a la precipitación y a la humedad relativa, las diferencias fueron mayores, sobre todo a escala estacional como también se visualizó en el estudio de Kim et al. (2019). Por ello, es importante considerar la incorporación de un mayor número de estaciones meteorológicas dentro de cada cuadrícula del Reanalysis para poder identificar el patrón espacial al que hacen referencia y eliminar los efectos de la topografía (Libanda et al., 2020).

\section{Conclusiones}

Del análisis realizado a ciudades costero fluviales y marinas de clima cálido, templado y frio (húmedos, de transición y seco) en Argentina, se obtuvieron buenas correlaciones en algunas escalas temporales 
para los parámetros meteorológicos del Reanalysis respecto de los datos observados. En la escala climática, los valores medios para todo el período de estudio (60 años) presentaron, en general, una buena estimación entre ambas bases de datos. La temperatura del aire tuvo buena correlación, con valores máximos de diferencias en las ciudades de clima cálido de hasta $2^{\circ} \mathrm{C}$. La humedad relativa tuvo un ajuste menor al $10 \%$ en todos los casos y la precipitación fue más exacta para los climas áridos y templados de transición. La única ciudad con diferencias significativas en todos los parámetros analizados fue Río Gallegos.

Del análisis estacional se obtuvo que la temperatura del aire no presentó, en general, buena correlación. La humedad relativa mostró mejor ajuste en invierno y otoño, excepto en Río Gallegos. Finalmente, la precipitación, al igual que en la escala climática, el ajuste fue mejor para los climas templados y áridos. Respecto de la escala interanual, los resultados presentaron errores mayores por lo cual se recomienda testear las series de datos para realizar el ajuste estadístico. Asimismo, los mejores ajustes fueron en la temperatura del aire y los de menor ajuste fueron en la humedad relativa.

El análisis mensual mostró mejores ajustes para la temperatura del aire. En la humedad relativa los ajustes fueron buenos, exceptos para las ciudades de clima cálido y húmedo, donde los valores originales no tuvieron buena correlación con los del Reanalysis. Finalmente, la precipitación tuvo una buena correlación. Con estos datos se generó una serie modelada con buenos ajustes mensuales para la temperatura del aire y la humedad relativa. Las precipitaciones fueron más complejas, no se logró identificar las estaciones secas en los climas cálidos húmedos, en especial en el caso de la ciudad de Concordia.

Los resultados obtenidos en este estudio sirven de base para comprender las diferencias que el Reanalysis tiene para la caracterización climática de ciudades costeras localizadas en distintos climas de Argentina. Este conocimiento es de vital importancia para diseñar un plan de manejo de los espacios urbanos destinados a la conservación de los recursos naturales y la mitigación de los efectos de la variabilidad climática sobre estos ambientes. Los mismos podrían ser de gran utilidad para que los tomadores de decisiones y los actores sociales mejoren su calidad de vida considerando los lineamientos del desarrollo sustentable.

\section{Agradecimientos}

Los autores desean agradecer al Consejo Nacional de Investigaciones Científicas y Técnicas (CONICET) y a la Universidad Nacional del Sur (UNS) por la financiación del presente estudio. Además, al Servicio Meteorológico Nacional (SMN, Argentina) y al Instituto Nacional de Tecnología Agropecuaria (INTA, Argentina) por el suministro de datos meteorológicos. Finalmente, agradecemos los datos del Reanálisis proporcionados por NOAA/ OAR/ESRL PSD, Boulder, Colorado, Estados Unidos a través de su sitio web: http://www.esrl.noaa.gov/psd/.

\section{Referencias}

Abatzoglou, J. T., \& Brown, T. J. (2012). A comparison of statistical downscaling methods suited for wildfire applications. International Journal of Climatology, 32(5), 772-780. https://doi.org/10.1002/ joc. 2312

Araya-Osses, D., Casanueva, A., Roman-Figueroa, C., Uribe, J. M., \& Paneque, M. (2020). Climate change projections of temperature and precipitation in Chile based on statistical downscaling. Climate Dynamics, 54(9), 4309-4330. https://doi.org/10.1007/s00382-020-05231-4

Boé, J., Terray, L., Habets, F., \& Martin, E. (2007). Statistical and dynamical downscaling of the Seine basin climate for hydro-meteorological studies. International Journal of Climatology: A Journal of the Royal Meteorological Society, 27(12), 1643-1655. https://doi.org/10.1002/joc.1602

Bustos, M. L., Ferrelli, F., Huamantinco Cisneros, M. A., Piccolo, M. C., \& Gil, V. (2016). Estudio preliminar del ajuste entre datos meteorológicos in situ y del Reanálisis (NCEP/NCAR) en distintos ambientes de la provincia de Buenos Aires, Argentina. Estudios Geográficos, LXXVII, 280, 335-343.

Bustos, M. L., Ferrelli, F., \& Piccolo, M. C. (2017). Estudio Comparativo de Tres Modelos Climáticos en Argentina. Anuário do Instituto de Geociências, 40(1), 34-43. http://dx.doi.org/10.11137/2017_1_34_43

Cannon, A. J., Sobie, S. R., \& Murdock, T. Q. (2015). Bias correction of GCM precipitation by quantile mapping: How well do methods preserve changes in quantiles and extremes?. Journal of Climate, 28(17), 6938-6959. https://doi.org/10.1175/JCLI-D-14-00754.1 
Comisión Económica para América Latina y el Caribe (CEPAL) (2018). Proyecto de primer informe regional sobre la implementación del Consenso de Montevideo sobre Población y Desarrollo (LC/CRPD.3/3), Santiago de Chile. Recuperado de https://repositorio.cepal.org/bitstream/handle/11362/43708/1/ S1800378_es.pdf

Collazo, S., Barrucand, M., \& Rusticucci, M. (2019). Summer seasonal predictability of warm days in Argentina: statistical model approach. Theoretical and Applied Climatology, 138(3-4), 1853-1876. https://doi.org/10.1007/s00704-019-02933-6

da Rocha Repinaldo, C. R., Müller, G. V., Martins Andrade, K. (2017). Patrones Atmosféricos Simulados en el clima presente y futuro asociados al Descenso de temperatura en el invierno en el sudeste de Sudamérica. Boletín geográfico, 39, 13-34.

Garreaud, R. (2000). Cold Air Incursions over Subtropical South America: Mean Structure and Dynamics, Monthly Weather Review, 128(7), 2544-2559. https://doi.org/10.1175/1520-0493(2000)128<2544:CA $\underline{\mathrm{IOSS}>2.0 . \mathrm{CO} ; 2}$

Gomes de Lima, J. A., \& Rodrigues Alcântara, C. (2019). Comparison between ERA Interim/ECMWF, CFSR, NCEP/NCAR reanalysis, and observational datasets over the eastern part of the Brazilian Northeast Region. Theoretical and Applied Climatology, 138(3-4), 2021-2041. https://doi.org/10.1007/ s00704-019-02921-w

Devers, A., Vidal, J. P., Lauvernet, C., Graff, B., \& Vannier, O. (2020). A framework for high-resolution meteorological surface reanalysis through offline data assimilation in an ensemble of downscaled reconstructions. Quarterly Journal of the Royal Meteorological Society, 146(726), 153-173. https://doi. org/10.1002/qj.3663

Eum, H. I., \& Cannon, A. J. (2017). Intercomparison of projected changes in climate extremes for South Korea: application of trend preserving statistical downscaling methods to the CMIP5 ensemble. International Journal of Climatology, 37(8), 3381-3397. https://doi.org/10.1002/joc.4924

Ferrelli, F., Brendel, A. S., Aliaga, V. S., Piccolo, M. C., \& Perillo, G. M. E. (2019). Climate regionalization and trends based on daily temperature and precipitation extremes in the south of the Pampas (Argentina). Cuadernos de Investigación Geográfica, 45(1), 393-416. https://doi.org/10.18172/cig.3707

Ferrelli, F., Bustos, M. L., Piccolo, M. C., Huamantinco Cisneros, M. A. \& Perillo, G. M. E. (2016). Downscaling de variables climáticas a partir del reanálisis NCEP/NCAR en el sudoeste de la provincia de Buenos Aires (Argentina). Papeles de Geografía, (62), 21-33. https://doi.org/10.6018/ geografia/2016/239051

Haylock, M. R., Cawley, G. C., Harpham, C., Wilby, R. L., \& Goodess, C. M. (2006). Downscaling heavy precipitation over the United Kingdom: a comparison of dynamical and statistical methods and their future scenarios. International Journal of Climatology: A Journal of the Royal Meteorological Society, 26(10), 1397-1415. https://doi.org/10.1002/joc.1318

Heo, J. H., Ahn, H., Shin, J. Y., Kjeldsen, T. R., \& Jeong, C. (2019). Probability distributions for a quantile mapping technique for a bias correction of precipitation data: A case study to precipitation data under climate change. Water, 11(7), 1475. https://doi.org/10.3390/w11071475

Hwang, S., \& Graham, W. D. (2013). Development and comparative evaluation of a stochastic analog method to downscale daily GCM precipitation. Hydrology and Earth System Sciences, 17(11), 44814502. https://doi.org/10.5194/hess-17-4481-2013

Jeon, S., Paciorek, C. J., \& Wehner, M. F. (2016). Quantile-based bias correction and uncertainty quantification of extreme event attribution statements. Weather and Climate Extremes, 12, 24-32. https://doi.org/10.1016/j.wace.2016.02.001

Kalnay, E., Kanamitsu, M., Kistler, R., Collins, W., Deaven, D., Gandin, L., Iredell, M., ... \& Joseph, D. (1996). The NCEP/NCAR 40-year reanalysis project. Bulletin of the American meteorological Society, 77(3), 437-472. https://doi.org/10.1175/1520-0477(1996)077<0437:TNYRP>2.0.CO;2

Kim, K. Y., Kim, J., Boo, K. O., Shim, S., \& Kim, Y. (2019). Intercomparison of precipitation datasets for summer precipitation characteristics over East Asia. Climate Dynamics, 52(5-6), 3005-3022. https:// doi.org/10.1007/s00382-018-4303-3 
Kistler, R., Kalnay, E., Collins, W., Saha, S., White, G., Woollen, J., Chelliah, M., ... \& Fiorino, M. (2001). The NCEP-NCAR 50-year reanalysis: monthly means CD-ROM and documentation. Bulletin of the American Meteorological society, 82(2), 247-268. https://doi.org/10.1175/1520-0477(2001)082<0247:TNNYRM $\geq 2.3 . \mathrm{CO} ; 2$

Libanda, B., Bwalya, K., Nkolola, N. B., \& Chilekana, N. (2020). Quantifying long-term variability of precipitation and temperature over Zambia. Journal of Atmospheric and Solar-Terrestrial Physics, 198, 105201. https://doi.org/10.1016/j.jastp.2020.105201

Martins Andrade, K. M., Müller, G. V., Cavalcanti, I. F. A. Fernández-Long, M. E. \& Bidegain, M.(2012): Avaliação de mudanças na frequência de sistemas frontais sobre o sulda América do Sul em projeções do clima futuro. Meteorologica, 37, 15-26.

Mesinger, F., DiMego, G., Kalnay, E., Mitchell, K., Shafran, P. C., Ebisuzaki, W., .. \& Shi, W. (2006). North American regional reanalysis. Bulletin of the American Meteorological Society, 87(3), 343-360. https:// doi.org/10.1175/BAMS-87-3-343

Miró, J., Estrela Navarro, M. J., Pastor, F., \& Millán Muñoz, M. (2009). Análisis comparativo de tendencias en la precipitación, por distintos" inputs", entre los dominios hidrológicos del Segura y del Júcar (1958-2008). Investigaciones Geográficas, (49), 129-157. https://doi.org/10.14198/INGEO2009.49.07

Miró Pérez, J. J. , Estrela Navarro, M. J., \& Olcina Cantos, J. (2015). Statistical downscaling and attribution of air temperature change patterns in the Valencia region (1948-2011). Atmospheric Research, 156, 189-212. https://doi.org/10.1016/j.atmosres.2015.01.003

Nacar, S., Kankal, M., \&Okkan, U. (2019). Statistical Downscaling of Monthly Mean Air Temperature Using NCEP/NCAR Re-analysis Data: A Case Study for the Eastern Black Sea Basin. En $3^{\text {rd }}$ International Conference on Advanced Engineering Technologies. Turkia. https://www.researchgate.net/profile/ Sinan_Nacar/publication/336374233

Piccolo, M. C., Merlotto, A. Bustos, M. L., Huamantinco Cisneros, M. A. \& García, M. A. (2017). Tormentas severas: ¿Cuán vulnerables son las costas del sur de la provincia de Buenos Aires (Argentina)? En A.V. Botello, S. Villanueva, J. Gutiérrez y J. L. Rojas-Galaviz (Eds.), Vulnerabilidad de las zonas costeras de Latinoamérica al cambio climático (pp. 283-296). Recuperado de https://ri.conicet.gov.ar/ handle/11336/129944

Romero, H. (2019). Urban Climates and the Challenge of Sustainable Development of Chilean Cities. En C. Henríquez y H. Romero (Eds.), Urban Climates in Latin America (pp. 207-256). Springer International Publishing. https://doi.org/10.1007/978-3-319-97013-4

Romero, P. E., González, M. H., Rolla, A. L., \& Losano, F. (2020). Forecasting annual precipitation to improve the operation of dams in the Comahue region, Argentina. Hydrological Sciences Journal, 1-10. https://doi.org/10.1080/02626667.2020.1786570

Rusticucci, M. M., \& Kousky, V. E. (2002). A comparative study of maximum and minimum temperatures over Argentina: NCEP-NCAR reanalysis versus station data. Journal of Climate, 15(15), 2089-2101. https://doi.org/10.1175/1520-0442(2002)015<2089:ACSOMA>2.0.CO;2

Sachindra, D. A., Ahmed, K., Mamunur Rashid, M., Sehgal, V., Shahid, S., \& Perera, B. J. C. (2019). Pros and cons of using wavelets in conjunction with genetic programming and generalised linear models in statistical downscaling of precipitation. Theoretical and Applied Climatology, 138(1-2), 617-638. https://doi.org/10.1007/s00704-019-02848-2

Seo, S. B., \& Kim, Y. O. (2018). Impact of spatial aggregation level of climate indicators on a nationallevel selection for representative climate change scenarios. Sustainability, 10(7), 2409. https://doi. org/10.3390/su10072409

Seth, A., Rauscher, S. A., Camargo, S. J., Qian, J. H., \& Pal, J. S. (2007). RegCM3 regional climatologies for South America using reanalysis and ECHAM global model driving fields. Climate Dynamics, 28(5), 461-480. https://doi.org/10.1007/s00382-006-0191-z

Takane, Y., Kikegawa, Y., Hara, M., \& Grimmond, C. S. B. (2019). Urban warming and future airconditioning use in an Asian megacity: importance of positive feedback. Climate and Atmospheric Science, 2(1), 1-11. https://doi.org/10.1038/s41612-019-0096-2 
Viggiano, M., Busetto, L., Cimini, D., Di Paola, F., Geraldi, E., Ranghetti, L., Ricciardelli, E \& Romano, F. (2019). A new spatial modeling and interpolation approach for high-resolution temperature maps combining reanalysis data and ground measurements. Agricultural and Forest Meteorology, 276, 107590. https://doi.org/10.1016/j.agrformet.2019.05.021

Xue, Y., Janjic, Z., Dudhia, J., Vasic, R., \& De Sales, F. (2014). A review on regional dynamical downscaling in intraseasonal to seasonal simulation/prediction and major factors that affect downscaling ability. Atmospheric research, 147, 68-85. https://doi.org/10.1016/j.atmosres.2014.05.001

Zhang, X. \& Yang, F. (2013). RClimDex (1.1) user manual. Retrieved from https://acmad.net/rcc/ procedure/RClimDexUserManual.pdf

Zhao, T., Guo, W., \& Fu, C. (2008). Calibrating and evaluating reanalysis surface temperature error by topographic correction. Journal of Climate, 21(6), 1440-1446. https://doi.org/10.1175/2007JCLI1463.1

Zhou, H., Aizen, E., \& Aizen, V. (2018). Constructing a long-term monthly climate data set in central Asia. International Journal of Climatology, 38(3), 1463-1475. https://doi.org/10.1002/joc.5259 